\title{
1 A Stochastic Model for Actin Waves in Eukaryotic Cells
}

2 Jifeng $\mathrm{Hu}^{1}$, Varunyu Khamviwath ${ }^{2}$, Hans G. Othmer ${ }^{3, *}$

31 School of Mathematics, University of Minnesota, Minneapolis, Minnesota, United States

4 of America

52 School of Mathematics University of Minnesota, Minneapolis, Minnesota, United States

6 of America

73 School of Mathematics and Digital Technology Center, University of Minnesota,

8 Minneapolis, Minnesota, United States of America

$9 \quad *$ E-mail: othmer@math.umn.edu

\section{Abstract}

${ }_{11}$ A stochastic model of spontaneous actin wave formation in eukaryotic cells that includes positive feedback 12 between the actin network and filament nucleating factors on the membrane is developed and analyzed.

13 Simulation results show that the model can produce a variety of actin network behavior depending on the

14 conditions. Actin spots of diameter about $0.5 \mu \mathrm{m}$ can be formed and persist for tens of seconds at low

15 actin concentrations, and these spots may either shrink and die or grow and develop into fully-developed

16 propagating waves. The model correctly captures the vertical profile of actin waves along line scans

17 through wave fronts, as well as the separation between the region enclosed by circular actin waves and

18 the external area. Our results show how the complicated actin behavior depends on the amounts and

19 state of various membrane molecules.

\section{${ }_{20}$ Author Summary}

${ }_{21}$ Locomotion of eukaryotic cells is a complex process that involves the spatio-temporal control and inte-

22 gration of a number of sub-processes, including the transduction of chemical or mechanical signals from

23 the environment, local and global modification of the cytoskeleton, and translation of the intra- and

24 extracellular signals into a mechanical response. In view of the complexity of the processes, understand-

25 ing how force generation and mechanical interactions with the surroundings are controlled in space and

26 time to produce cell-level movement is a major challenge. Recent experimental work has shown that a 
1 variety of actin waves propagate within cells, both under normal conditions and during re-building of the

2 cytoskeleton following its disruption. Controlled disruption and re-building of the actin network has led

3 to new insights into the key components involved in actin waves, and here we develop a stochastic model

4 that can qualitatively and quantitatively describe the dynamical behavior of such waves.

\section{${ }_{5}$ Introduction}

6 Cell locomotion is essential for numerous processes, including early development, angiogenesis, tissue 7 regeneration, the immune response, and wound healing in multicellular organisms, and plays a very 8 deleterious role in cancer metastasis in humans. Locomotion involves the detection and transduction 9 of extracellular chemical and mechanical signals, integration of the signals into an intracellular signal, 10 and the spatio-temporal control of the intracellular biochemical and mechanical responses that lead 11 to force generation, morphological changes and directed movement [1]. Controlled deformation and 12 remodeling of the cytoskeleton, which comprises actin filaments, intermediate filaments, and microtubules, 13 are essential for movement. The biochemical control processes, the microstructure of the cytoskeleton, 14 and the formation and dissolution of adhesion sites are coordinated at the whole-cell level to produce

15 the forces needed for movement. While a qualitative description of many of the constituent biochemical 16 steps in signaling and force generation are known, an integrated quantitative description of whole cell 17 motility is still a distant goal. To achieve that requires a mathematical model that links molecular-level 18 processes with macroscopic observations on forces exerted, cell shape, and cell speed because the large19 scale mechanical effects cannot be predicted from the molecular biology of individual steps alone. This is 20 best done in steps, using observations on successively more complex systems with the aim of developing ${ }_{21}$ whole cell models. Here we do this for a relatively simple system that nonetheless displays a variety of 22 interesting dynamical behavior relevant to cell motion in general.

23 In the absence of directional signals neutrophils and Dictyostelium discoideum (Dd) explore their envi24 ronment randomly $[2,3]$, and thus the intracellular biochemical networks that control the mechanics must 25 be tuned to produce signals that generate this random movement. In neutrophils three Rho GTPases 26 Cdc42, Rac and RhoA - which are activated by Ras, control three pathways that lead to the assembly of 27 filopodia [4], the formation of lamellipodia [5,6], and the contraction of the F-actin networks, respectively. 28 In mammalian cells activation of RhoA leads to inactivation of MLCPase, an inhibitor of myosin con- 
1 traction [7], and thereby to contraction. Rac activates factors such as ezrin, which localizes at points of 2 actin fiber attachment to the membrane and facilitates nucleation of actin polymerization by regulating 3 Arp2/3 [8]. The balance between the RhoA and Rac pathways determines whether dendritic network 4 formation or bundling of F-actin dominates. In the absence of directional signals the competition between 5 them can lead to complex patterns of traveling actin waves in the cortex in both cell types $[2,3,9,10]$. The 6 waves are typically closed and of varying shape, and they propagate by treadmilling, as shown by actin recovery after bleaching [11]. Myosin-IB, which links the actin network to the membrane [12], is found 8 at the front of a wave, and the Arp2/3 complex and a dense dendritic network are found throughout the wave. Coronin inhibits filament nucleation and indirectly regulates cofilin activity via dephosphorylation [13] throughout a wave, and cortexillin, which is found where PIP3 is low, organizes actin filaments into anti-parallel bundles. This type of actin wave is commonly observed in eukaryotic cells, and can arise under many physiological conditions $[2,14,15]$. It has been suggested that cells may utilize such waves in locomotion $[2,15]$, or to search for phagocytizing objects on the substrate surface [16].

In the presence of a chemotactic signal the cells must orient properly, which means the dynamical 15 system controlling the mechanics must respond to the bias. It is known that PTEN, which converts $16 \mathrm{PIP}_{3}$ to $\mathrm{PIP}_{2}$, is a major regulator of migration during chemotaxis in both Dd and neutrophils $[17,18]$. 17 Activated PI3K is increased at the site of signal reception and PTEN localizes at the lateral and posterior 18 regions of migrating cells. Myo-II, and hence contraction, is localized at the posterior end of migrating 19 neutrophils and Dd [19]. Whether PTEN controls myosin-II localization is not known, but it is known that 20 PTEN localizes at the side and the rear prior to myosin-II localization [20]. This suggests that PTEN may ${ }_{21}$ be involved in a positive feedback loop in which contraction enhances accumulation of PTEN and myosin22 II [20]. However, PTEN is not the sole controller of myosin localization, for it still localizes in $p^{-} n^{-}$ 23 cells, and this may involve the cGMP pathway in Dd or the RhoA/Rock pathway in neutrophils [21]. An 24 integrated model of the wave dynamics in the absence of signals, as well as in the presence of a directional 25 bias, is needed to explain the foregoing observations and others, including the fact that PI3K-null Dd cells 26 still chemotact in strong gradients, but their speed is reduced [22,23], and the fact that image analysis 27 shows that there is no temporal correlation between contraction and pseudopod extension [24].

28 The best characterized actin waves are those that arise during re-construction of the actin cytoskeleton 29 following treatment of cells with latrunculin, which leads to depolymerization of actin networks [9]. These 30 waves only arise at those parts of the cell membrane in contact with a substrate, and thus membrane- 
1 surface interaction is essential. Actin structures in the shape of spots initially form on the ventral 2 membrane of the substrate-attached $(\mathrm{SA})$ cell, and then propagates radially in roughly circular shape 3 with a prominent wave front and a decaying wave back [14], as seen in Fig. 1. The protein components 4 involved in the wave have been identified using confocal microscopy and total internal reflection fluo5 rescence microscopy (TIRF), which targets labeled species within a thin region near the cell-substrate 6 interface (usually less than $200 \mathrm{~nm}$ ). When combined with fluorescence recovery after bleaching (FRAP) experiments, TIRF imaging indicates that the wave on the membrane propagates not via direct transport of existent filaments, but rather, through de novo polymerization at the leading edge of the wave and in situ depolymerization at the trailing edge [14]. Imaging of the three-dimensional actin waves shows that continual growth of the actin network at the membrane pushes the network upward into the cytoplasm as shown in the schematic in Fig. 2(top). It also shows that the maximum network height occurs at the boundary between domains of high and low PIP3 levels, as shown in Fig. 2(bottom). Thus the PI3K13 driven component of the dynamics described in detail later lags the leading edge, and other processes dominate there.

Imaging of labeled components has identified the critical actin-binding proteins (ABPs) involved in 16 network re-construction [9]. The actin network in the wave is believed to be dendritic, similar to that in 17 the lamellipodium, due to the high concentration of Arp2/3 complexes measured. The Arp2/3 complex 18 is composed of seven subunits, and can be activated by binding to nucleation-promoting factors (NPF's), 19 monomeric actin (G-actin) and existing filaments. This interaction can lead to the formation of new 20 filaments, in which Arp2/3 complex caps the pointed end and attaches it to the mother filament. In 21 latrunculin-treated Dictyostelium cells, myosin-IB (MyoB), a single-headed motor molecule that binds to 22 the membrane and to actin filaments in the cortex, is localized at the wave front, close to the membrane.

${ }_{23}$ The scaffolding protein CARMIL is probably recruited to the wave front by MyoB, and acts as an ${ }_{24} \mathrm{NPF}$ for the Arp2/3 complex. In addition to CARMIL, other NPF's, such as the hetero-pentameric 25 WAVE/SCAR complex found in neutrophils [2] or WASP and SCAR in Dictyostelium [25], may activate

26 Arp2/3. However, to activate Arp2/3, NPF's must first be activated on the membrane by binding to ${ }_{27}$ phospholipids (phosphatidylinositol-4,5-bisphosphate, $\mathrm{PIP}_{2}$ and/or phosphoinositol-3,4,5-trisphosphate, ${ }_{28}$ PIP3) and small GTPases (Rac and Cdc42). It is also observed that coronin, which is bound to filaments 29 at the top and the back of the wave (cf. Fig. 2(top)), probably destabilizes the network by removing 30 Arp2/3 from a branch junction, thus exposing the pointed end to depolymerization [26]. A schematic of 
1 these interactions suggested in [9] is shown in Fig. 3.

2 The signaling cascades that initiate and sustain the actin waves are not well-defined as yet, but a 3 skeleton of the network has been established, and there are several distinct phases involved. The fact 4 that waves are only initiated on membrane that is attached to a surface is evidence for an unknown 5 linkage to substrate adhesion. Investigation of the relationship between wave initiation and membrane 6 adhesion to the substratum or extracellular matrix (ECM) has shown that a wave of activated integrin receptor trails the F-actin both temporally and spatially [27]. Actin filaments in the wave sequentially recruit various adhesion adaptor proteins to the cortex-membrane interface, and trigger integrin activation and interaction with the ECM. Interruption of this interaction inhibits actin wave expansion, which suggests that there is a positive feedback from integrin-mediated membrane adhesion to F-actin network formation and wave propagation. This may arise via the integrin-PI3K-Rac signaling cascade, since integrin-mediated adhesion domains are centers for actin polymerization. While the details of these interactions are not completely understood, experimental evidence shows that focal adhesion kinase (FAK) associated with a newly formed integrin domain could bind N-WASP and Arp2/3 directly, which promotes actin polymerization at nascent lamellipodia [28]. While Dd does not have integrins, it has integrin homologs [29].

Actin spots, which are the precursors of actin waves, are often found at sites of clathrin-mediated endocytosis, which suggests that incipient endocytosis may provide early filament components for the 19 actin waves [30]. However this is not the only source, since some actin spots are initiated at clathrin-free sites. Filament precursors may also come from unspecified residual actin network on the membrane, 21 or unbranched filaments nucleated by nucleators such as formin and ponticulin, but sustained F-actin 22 nucleation requires continual activation of NPF's by phospholipids. For the WAVE/Scar complex, the ${ }_{23}$ phospholipid is $\mathrm{PIP}_{3}$, the product of PI-3-kinase-mediated phosphorylation of $\mathrm{PIP}_{2}$. TIRF images reveal 24 that the membrane associated with a wave front and its inner area is enriched with $\mathrm{PIP}_{3}$. $\mathrm{A}$ positive 25 feedback circuit of Ras/PI3K/F-actin was thought to be involved in initiation of actin network assembly 26 at the leading edge of chemotactic cells [31], since PI3K phosphorylates $\mathrm{PIP}_{2}$ to $\mathrm{PIP}_{3}$, and the latter leads 27 to NPF activation and consequent Arp2/3-driven actin network formation. PI3K and $\mathrm{PIP}_{3}$ are essential ${ }_{28}$ for actin wave formation and propagation, since all wave activity ceases when cells are treated with a 29 PI3K-inhibitor (LY294002), and waves recover after LY294002 wash-out [11]. However, the correlation 30 between $\mathrm{F}$-actin and $\mathrm{PIP}_{3}$ is more complicated, because $\mathrm{PIP}_{3}$ usually lags behind the F-actin peak [3] 
1 , and the F-actin peak occurs at the steepest gradient [16]. A recent experiment suggests that $\mathrm{PIP}_{3}$

2 may down- regulate some Rho-family GTPases by activating relevant GAP's, which accelerate nucleotide

3 hydrolysis of GTP-bound GTPases [32]. The complex F-actin signaling network on the membrane is 4 summarized in Fig. 4.

5 In the following section we discuss existing models for actin wave generation, and in subsequent 6 sections we discuss our model in detail and present computational results from stochastic simulations of 7 the model.

\section{\& Existing mathematical models}

9 A number of mathematical models have been proposed to explain different aspects of actin waves in eu-

10 karyotic cells. It is well known that two-component reaction-diffusion systems of either Fitzhugh-Nagumo

11 (FN) or activator-inhibitor type can exhibit the formation and propagation of waves and the transitions

12 between actin patterns observed experimentally, and these dynamics have served as the starting point

13 for several phenomenological models. It is observed experimentally that Hem-1, which is one component

14 in the WAVE complex, localizes to the leading edge of actin waves in neutrophils [2]. Furthermore, actin

15 stimulates its own assembly, and actin filaments are involved in removing the Hem-1 complex from the

16 membrane. A heuristic model for the observed Hem-1 waves was proposed in which membrane-attached

17 Hem-1 is self-activating, it stimulates actin filament formation, and the F-actin nucleated by Hem-1 re-

18 leases Hem-1 from the membrane and thus inhibits the wave. Since the model is not derived from specific

19 molecular interactions the parameters in it are not experimentally measurable.

20

A more complex phenomenological model was developed by Whitelam et al., who described the dynamics of actin fibers with modified FN equations [33]. The actin fiber density serves as the analog of the membrane voltage in an FN model, while the corresponding FN inhibitor is assumed to degrade the actin network. Isotropic filament nucleation by the Arp2/3 complex at the barbed end is assumed, and the evolution of filament orientation is described by a separate evolution equation. With properly chosen parameters, the model predicts that the initial actin spot can transform from static spot to moving spot and subsequently to traveling waves, but again, the model is phenomenological and comparison with molecular mechanisms is difficult.

Recently, Carlsson proposed a detailed molecule-based stochastic model of actin wave formation and 
1 propagation [34]. The cytoplasmic part of the F-actin wave is assembled based on the known processes

2 in actin network formation, which include filament branching, severing, capping, and end-wise polymer-

3 ization and depolymerization. The author concluded that wave propagation depends on three major

4 processes: auto-catalytic polymerization of F-actin, random filament orientation, and slow recruitment of

5 NPF from the cytoplasm to the membrane. However there are restrictions imposed by the assumptions,

6 for instance, any actin cluster whose nearest distance from the membrane is larger than $60 \mathrm{~nm}$ is assumed

to disappear and is removed from the simulation. Thus the model can give some insight into the dynamics

near the surface, but cannot reproduce the full height of the network. In addition, the propagation of

9 the waves is driven in part by diffusion of the network along the membrane.

To date, none of the existing models includes coupling between F-actin polymerization and membranebound protein activities via a positive feedback loop, despite experimental evidence which suggests that 12 such feedback is important for expansion of the wave $[9,27]$. In light of this evidence we propose a stochastic model of actin wave initiation and propagation that incorporates the major components of the 14 actin filament polymerization machinery and a positive feedback loop between F-actin polymerization 15 and membrane-bound NPF's. In our model NPF exists in one of three inter-convertible states on the 16 membrane: a native inactive state $(\mathrm{NPF})$, an activated state $\left(\mathrm{NPF}^{*}\right)$ and a recovery state $\left(\mathrm{NPF}^{* *}\right)$. ${ }_{17} \mathrm{NPF}$ is activated by free filament barbed ends in close proximity to the membrane, the activated state ${ }_{18} \mathrm{NPF}^{*}$ is converted to the recovery state $\mathrm{NPF}^{* *}$ after participation in filament branch nucleation, and $19 \mathrm{NPF}^{* *}$ slowly reverts to the native inactive state. It is known that NPFs can diffuse on the membrane, 20 and a major conclusion from our analysis is that the spread of de novo filament nucleation above the ${ }_{21}$ basal level is driven by lateral diffusion of $\mathrm{NPF}^{*}$ that is activated locally by F-actin, and this drives 22 expansion of actin waves. This differs from a recent model [34] wherein network expansion depends on 23 filament elongation in the direction parallel to the plane of the membrane. Our simulation results agree 24 qualitatively with the characteristics and various behaviors of actin waves in vivo, and in particular, show 25 that localized, highly-branched actin spots emerge at sites where NPF is activated. At low levels of NPF 26 activation an actin spot either grows for a short time and then dies out, or it forms an expanding wave.

${ }_{27}$ The fate of an actin spot is determined by two competing processes: NPF activation by NPF-stimulated

${ }_{28}$ F-actin nucleation and conversion of $\mathrm{NPF}^{*}$ into $\mathrm{NPF}^{* *}$ after F-actin nucleation. Mobile actin spots can

29 form randomly, and adjacent actin spots may develop into circular actin waves within the region over

30 which a wave has passed. This continual generation of actin spots and their maturation into waves agrees 
1 with the observations in substrate-attached cells [2]. Our results also show that the dynamics are those of

2 an excitable medium, in that when multiple wave fronts collide they annihilate each other and eventually

3 die out, as observed in vivo [9]. An intriguing result of our model is that when a wave propagates to

4 the border of a region in which indigenous nucleation sites are present, the wave stalls and eventually

5 decays, which replicates the in vivo behavior when it reaches to the border of the SA portion of the

6 cell membrane [9]. The simple positive feedback loop between F-actin and NPF in the current model

7 can easily be extended by incorporating details of signaling networks such as the Ras/PI3K/Factin and

8 integrin/PI3K/Rac pathways as they become known, so as to more realistically describe the coupling

9 between membrane adhesion and F-actin waves.

\section{${ }_{10}$ Network Dynamics}

11 To facilitate understanding of the computational results presented in the following section, we describe

12 the evolution of the system from initiation of waves to the propagation stage. The major reaction steps

13 are depicted in Fig. 5, and the governing equations, the parameters used in the simulations, and a

14 description of the stochastic simulation algorithm, are given in the Materials and Methods section.

15

Initially G-actin, Arp2/3, coronin and capping protein (CP) are distributed uniformly in the cytosol,

16 where they diffuse freely, whereas NPF's diffuse on the membrane. Indigenous nucleation sites at which

17 filaments can nucleate are fixed on the membrane, but this is energetically unfavorable and hence only

18 occurs at a low basal rate. These nucleation sites could be viewed as membrane-anchored filament

19 nucleators such as formin or ponticulin. We call the filaments nucleated at these sites backbone filaments,

20 to contrast them with the branched filaments initiated on backbone filaments by Arp $2 / 3$ complex. The

${ }_{21}$ barbed end of a backbone filament is attached at the nucleation site, perhaps by Myo-IB ( $c f$. Fig. 3) and

22 is able to elongate until CPs cap the barbed end and free nucleation sites for a new round of backbone

23 filament nucleation. We assume that backbone filament nucleation requires free nucleation sites, but not

24 NPF activation, and thus a basal level of F-actin nucleation and polymerization is always present on the

25 membrane. In the experimental context this only occurs in the SA portion of the membrane.

26 To initiate the dynamics, a small number of NPF's are activated locally to mimic the signaling

27 transduced from the SA portion of the membrane. Activated NPF (NPF*) then recruits Arp2/3 and

28 G-actin sequentially onto the membrane and forms a complex, which generates a filament branch upon 
1 binding to either a backbone filament or a previously-formed branch filament, either of which is called a mother filament. After nucleation of a new branch, Arp2/3 remains attached to the pointed end of the new filament until coronin binds and subsequently releases Arp2/3 from it, whereas NPF* released from

4 the daughter filament is converted into $\mathrm{NPF}^{* *}$, which slowly recovers to the inactive NPF state. NPF is activated into $\mathrm{NPF}^{*}$ by a free barbed end of a branched filament when the latter is within a threshold 6 distance $\left(L_{\text {nucl_zone }}\right)$ from the membrane. The simplified network involving feedback between barbed ends and NPF's is summarized in Fig. 6.

8

We assume that all filaments are vertically aligned, with the barbed ends facing the membrane. 9 Filaments do not diffuse or move in directions parallel to the membrane plane, but do undergo vertical 10 shifts due to elongation at the barbed end abutting the membrane. All filaments associated to the same nucleation site via Arp2/3-mediated branching constitute a local actin cluster (LAC). Filaments in a LAC are assumed to interconnected, which may be due to the tightness of inter-filament space or cross-linkage all filaments are tethered.

\section{${ }_{16}$ Results}

\section{${ }_{17}$ Actin wave generation and propagation}

We show in what follows that the stochastic model described above, which comprises the actin assembly 
1 an expanding wave, as seen in the snapshot of wave dynamics at 5 seconds in Fig. 7 . If we were to

2 initiate a wave in the center of a larger domain, the result would be an approximately circular wave that 3 propagates radially outward (See online video S3).

$4 \quad$ The wave initiated at the corner travels at a speed between 0.1 and $0.2 \mu \mathrm{m}$ per second, and the F-actin 5 density behind the wave gradually decays, thereby producing a relatively stable profile about 1-2 $\mu \mathrm{m}$ in 6 width. A circular wave separates the underlying membrane into three regions: the membrane beneath the wave, the area enclosed by the wave, and the outside area ahead of the wave. Though it is difficult to see 8 in the figure, both the interior and exterior regions are populated with small amounts of actin filaments 9 that arise via spontaneous filament nucleation activity at the nucleation sites. However, these two regions 1. differ in their molecular composition in that a large proportion of NPF on the interior membrane is the 11 recovery form $\mathrm{NPF}^{* *}$, whereas in the exterior region the NPF is in the native inactive state, as will be 12 shown later. When the wave reaches the boundary it decays slowly because the interior does not recover 13 fast enough to facilitate reversal of the wave. Following passage of a wave the dynamics take one of two 14 forms. In one form, the entire membrane is covered with a low level of F-actin generated by spontaneous 15 nucleation at distributed nucleation sites. Alternatively, some free barbed ends of branched filaments 16 remain following passage of the wave and serve as sites for the initiation of new waves, as will be shown 17 later.

${ }_{18}$ Further details of wave propagation can be understood quantitatively by examining a cross-section 19 of a propagating wave. An example of the time-evolution in Fig. 8 shows the outward propagation 20 of a roughly unimodal shape of F-actin along the diagonal line from the initial NPF activation site at 21 the left lower corner. By tracking the wavefront location we estimate an average speed slightly above ${ }_{22} 0.13 \mu \mathrm{m} / \mathrm{s}$. One sees that the wave front is steeper than the back, reflecting the rapid increase in 23 filament density due to the positive feedback loop. The basal level of F-actin ahead of the wave is 24 small compared to that within the wave, and the area well behind the wave also relaxes to a basal level 25 of F-actin corresponding to spontaneous filament polymerization at nucleation sites. Since continual ${ }_{26}$ F-actin polymerization requires supplies of new filament barbed ends mediated by activated NPF*, an ${ }_{27}$ examination of the NPF composition across the wave front sheds light on the important components ${ }_{28}$ in wave formation. Comparison of the three NPF profiles in Fig. 9 with the F-actin profile across the 29 membrane at 30 seconds in Fig. 8 reveals different NPF activities in different regions. The region outside

30 the wave, beyond $4 \mu \mathrm{m}$ shown in both figures, is devoid of Arp2/3-mediated branched filament nucleation 
1 due to the lack of activated $\mathrm{NPF}^{*}$ there, but has the potential for filament nucleation due to the presence

2 of a large amount of inactive NPF (blue line). Within the steep leading edge of the wave, which is around

$3 \quad 3.5-4 \mu \mathrm{m}$, the membrane is populated with a low level of activated $\mathrm{NPF}^{*}$, while behind the wave, between

42.5 and $3.5 \mu \mathrm{m}$, most of the NPF is in the recovery state (green line). There is little $\mathrm{NPF}^{*}$ in this region,

5 hence little branched filament nucleation by $\mathrm{NPF}^{*} /$ Arp2/3/actin complex, and this leads to the decay

6 of F-actin behind the peak. The region well behind the peak has some NPF that has recovered, but the

7 majority of the NPF is in the recovery state. Where there is some NPF in the inactive state, any residual

8 free barbed ends of branched filament could activate it and thus generate catalytic filament branching

9 locally. This is why one sometimes sees a new round of wave initiation and propagation long after the 10 wave has passed - an example of this will be shown in later section on colliding waves.

Since TIRF imaging only captures the density of labeled actin within $\sim 100-200 \mathrm{~nm}$ of the surface it 12 does not reflect the entire structure and evolution of the waves. All previous mathematical models of 13 actin waves only reproduce the F-actin within the range of TIRF imaging, but the optimized simulation 14 algorithm we use enables us to explore the entire wave structure. A stochastic realization of the entire 15 wave structure as it evolves, from which the previous images were extracted, is shown in Fig. 10.

This example shows clearly that an actin filament cluster grows rapidly where NPF's are activated.

\section{Determinants of the wave speed}

The current model not only produces propagating waves qualitatively similar to the experiments, but also permits detailed quantitative investigation to determine which processes regulate the propagation speed and the characteristic length scales of the wave. As indicated earlier, the leading edge is driven by $\mathrm{NPF}^{*}$ diffusion, and the rapid polymerization there is due to positive feedback between free barbed ends and activated NPF*. The propagation speed of a wave front is a complicated function of multiple inputs, such as the half life of $\mathrm{NPF}^{*}$ diffusion on the membrane prior to its association with an Arp2/3 complex, the strength of positive feedback between F-actin and NPF's, and the G-actin concentration. 
1 Parametric studies of the current model indicate that increased NPF diffusion, elevated spontaneous

2 filament nucleation, and amplified monomer concentration all lead to increases in the wave speed.

3 In light of the effects of latrunculin on the network, it is particularly important to understand how 4 the G-actin concentration influences the wave dynamics. It is observed in vivo that small and relatively

5 stationary actin spots first appear on the membrane in the early phase of F-actin reassembly when the 6 monomer-sequestering drug latrunculin is washed away. The free polymerizable G-actin is presumed 7 to be low in the cytosol at that stage since latrunculin gradually releases the bound G-actin following 8 washout. The underlying mechanism leading to spots is not established, but one possibility is that the stochastic membrane-ECM interaction excites local signaling on the membrane, leading to downstream actin polymerization [35]. In the following numerical experiment, we randomly choose nine membrane patches for NPF activation with a G-actin level as low as $0.1 \mu \mathrm{M}$. Only four of nine membrane spots give rise to detectable actin networks, as can be seen in Fig. 11. The TIRF density of F-actin is low, and more interestingly, it takes up to 50 seconds to develop actin spots $\sim 0.5 \mu \mathrm{m}$ in diameter, which suggests that they are essentially stationary.

JFH modify later We also did a parametric study of the dependence of wave speed on monomer concentrations. For each actin concentration, twenty realizations of the stochastic simulation were generated for quantification of the mean wave speed. As shown in Fig. 12, at low concentrations the mean speed is approximately linearly related to the actin concentration, whereas at high concentrations the speed approaches a saturation value. At low G-actin concentration the generation of barbed ends is low, and accordingly NPF activation is low, which weakens the wave propagation. At the other extreme of high G-actin concentration the barbed-end generation by $\mathrm{NPF}^{*}$-Arp2/3-actin complex may saturate as $\mathrm{NPF}^{*}$ is consumed and depleted locally, i.e., the positive feedback between F-actin and NPF saturates. In that regime a higher G-actin level will not elevate NPF activation further, and thus the speed will reach a plateau value.

\section{${ }_{25}$ Determinants of the wave shape} Two additional experimentally-measurable quantities are the width and density of the wave, which conof various NPF states and downstream F-actin in Fig. 8 and 9 shows that the rapid increase in network 
1 and its subsequent consumption by filament branch generation determines the width of the wave from the

2 leading edge to the peak, whereas the maximal density and height of the wave is determined by both of elongation rate at the barbed ends and duration of local filament nucleation. In contrast to the wave front, 4 the wave back lies over the $\mathrm{NPF}^{*}$-depleted membrane, where the supply of Arp2/3-mediated filament generation is diminished. In addition, barbed end capping and fast depolymerization of filament pointed ends exposed by coronin-mediated Arp2/3 removal causes the network to shrink from the cytoplasmic side. Therefore, the rapid action of coronin in removing Arp2/3 from filament branches, together with fast depolymerization at pointed ends, will reduce the width of the wave back. An increase in the CP concentration also accelerates filament turnover and leads to a decrease in the width of the wave back. At very high CP concentrations propagation can be blocked, since CP's also cap barbed ends at the leading edge, thereby weakening the positive feedback loop between F-actin and NPF. In summary, the wave 12 density and height are determined by the elongation rate at the barbed end and the NPF* consumption 13 rate, whereas the width of the wave back is primarily controlled by the pointed-end depolymerization 14 rate.

\section{${ }_{15}$ Wave annihilation and reformation at multiple sites}

16 One characteristic of actin waves on SA membranes is that they annihilate each other when they collide.

${ }_{17}$ Two processes in the current model produce such wave behavior: positive feedback of F-actin polymer18 ization from NPF activation at the wave front, and reduction of filament polymerization at the wave 19 back, due primarily to the slow recovery of NPF**. Simulations shown in Fig. 13 confirm this - there 20 four wave fronts generated at the four corners of the domain expand toward each other, and when they 21 collide they annihilate each other.

Nonetheless, some actin spots may survive in the wake of waves that collide. These spots are transient, they propagate in random directions, and they either disappear completely or gain sufficient strength to become precursors of new waves, as shown in Fig. 14. At 50 seconds into this simulation, two disconnected actin clusters move in different directions at the lower left corner, and later evolve into traveling F-actin arches. There us another isolated actin cluster at the upper right corner which develops into broken waves. either annihilate each other or form a new wave front that moves into a previously-undisturbed region of the membrane. Examination of the distribution of NPF at the onset of these transient actin spots 
1 shows widespread recovery of $\mathrm{NPF}^{* *}$. These NPF's, which can then be activated locally by residual free

2 barbed end in actin spots, can serve to trigger the catalytic F-actin polymerization through the positive

3 feedback of F-actin on NPF activation, as depicted by the distribution of various NPF states in Fig. 15.

4 JFH: comments on other panels?

\section{Standing waves}

6 It has been observed in vivo that the waves come to a halt when they reach the lateral cell border [14].

7 Frequently it may push the cell membrane forward before it retracts, but the wave cannot propagate

8 very far along unattached membrane. There are two possible explanations for this. Firstly, the positive

9 feedback of F-actin to NPF activation may require membrane attachment to the ECM, and while activated

10 NPF can diffuse to the unattached membrane, it cannot sustain its own activation due to the lack of

11 positive feedback. As a result, sustained F-actin polymerization is limited to the SA portion of the

12 membrane. Alternatively, other signaling proteins at the unattached membrane might actively inhibit

13 the positive feedback NPF experiences at the attached membrane, and this may stop propagation of a

14 wave across the unattached membrane. Experimental evidence shows that the membrane exterior to a

15 closed wave is occupied by PTEN, and the PTEN area may grow and push the wave backward, since

${ }_{16}$ PTEN converts $\mathrm{PIP}_{3}$ to $\mathrm{PIP}_{2}$, thus inhibits NPF activation.

To explore the wave behavior when it encounters the unattached membrane, we test our model on a 18 membrane comprised of two areas: a cental disk in which the positive feedback between F-actin and NPF 19 is active, and a surrounding area where barbed ends cannot activate NPF. The resulting dynamics of a 20 wave are displayed in Fig. 16. Note that when the wave hits the boundary it extends into the inactive ${ }_{21}$ area for a short distance of order $0.1-0.3 \mu \mathrm{m}$, which is determined by the distance over which an activated 22 NPF diffuses before binding with Arp2/3 correct ??. The other intriguing feature is that the wave can 23 persist as a standing wave at the border between the active and inactive regions of the membrane. We 24 propose that a continual supply of NPF from inactive regions to the active regions via diffusion sustains the wave at the border. If the inactive region expands at the cost of active region shrinkage, the NPF supply from outside to inside of the wave might lead the standing wave to travel inwardly as the reversible wave observed in experiments [36]. 


\section{The role of the recovery state}

2 One conclusion from our model is that the decay at the back of the wave results from the combination of

3 barbed-end capping by CP's, fast depolymerization at exposed pointed ends, and a lack of new filament

4 generation due to local depletion of $\mathrm{NPF}^{*}$ and slow recovery of $\mathrm{NPF}^{* *}$. Of these, the lack of $\mathrm{NPF}^{*}$ in

5 the negative feedback on F-actin polymerization at the wave back is a dominant factor. This is shown

6 by the fact that waves become transition waves rather than solitary waves, i.e., the waves propagate 7 outward but the network in the back of the wave does not decay, when the recovery rate of $\mathrm{NPF}^{* *}$

8 to NPF is increased. The extreme case of this arises when the recovery state is eliminated and $\mathrm{NPF}^{*}$ 9 is converted directly to NPF after its participation in filament generation. The dynamics of the two10 NPF-state model is illustrated in Fig. 17, which demonstrates that a slowly-recovering NPF** state is 11 critical for the observed wave structure. In accordance with this prediction, it is observed experimentally 12 that fluorescence recovery after photo-bleaching of the WAVE protein, one type of NPF, is slow in the 13 lamellipodium [37], and individually-tracked WAVE proteins are frequently found to detach from the 14 membrane or incorporate into the actin network after filament generation [38]. Thus the recovery state 15 in our model could be a stand-in for NPF that detaches from the membrane and then re-bind to the 16 membrane slowly. The simulation result with reversible binding of NPF to the membrane explicitly 17 considered can be seen in Fig. 18, which shows no significant difference from that of the model with a 18 third state as depicted in Fig. 7. However, the computation cost of the model with third NPF state is 19 half of that with explicit NPF detachment from the membrane.

\section{${ }_{20}$ Discussion}

${ }_{21}$ We propose a stochastic model for the evolution of actin waves observed in many cells, both under drug22 treated and physiological conditions. These waves involve dendritic actin networks that are initiated 23 on membranes that adhere to a substrate. The model links cytoplasmic actin polymerization to mem24 brane adhesion via proteins called NPFs. Currently, we assume fixed filament nucleation sites uniformly 25 distributed on the membrane, which could be membrane-bound filament nucleators such as formin or 26 ponticulin [39]. In light of experimental evidence for the existence of a positive feedback for filament 27 polymerization at the cortex-membrane interface, we introduce a NPF-free-barbed-end positive feedback ${ }_{28}$ loop, where free barbed ends activate membrane-bound NPF. We suppose that there is a low level of 
1 spontaneous filament nucleation at sites on the membrane, and these generate backbone filaments that 2 provide docking sites for Arp2/3-mediated filament branching. The local filament branching is further 3 strengthened by a positive feedback between NPF activation and free barbed ends. The actin assembly 4 machinery in the model comprises a minimal set of proteins - in addition to NPF - that can generate 5 a dynamically evolving actin network: Arp2/3, which nucleates branches and caps pointed ends on the

6 branch, coronin, a factor that contributes to Arp2/3 removal from the network and pointed-end exposure, and CP, a barbed-end capping protein that prevents polymerization. In this minimal model, we predict 8 that the formation and propagation of actin waves can be initiated with a local perturbation that activates sites on the membrane, and numerical experiments show that waves annihilate when they collide, and travel at speeds strongly depending on the actin concentration.

The simulation results show that the model is able to produce a variety of actin network behavior depending on the conditions. Actin spots of diameter about $0.5 \mu \mathrm{m}$ can be formed and persist for tens of seconds at low actin concentrations, These actin spots are dynamic, and are capable of migration, merging, and growth and vanishing. At high actin concentrations circular waves form at NPF activation sites and travel at 0.1-0.2 $\mu \mathrm{m}$ per second when fully developed. Transient mobile actin spots may either shrink and die or grow and develop into new rounds of coherent propagating waves. Our results show how the complicated actin behavior depends on the amounts and state of various membrane molecules.

The model also correctly captures the vertical profile of actin waves along line scans through wave fronts and the separation between the region enclosed by circular actin waves and the external area.

The decay of the wave back is caused by the slow recovery of NPF, which becomes NPF** upon branch creation. This slow recovery of NPF, which becomes NPF** upon branch creation, leads to exhaustion of active NPF and the decay of the wave back. In circular actin waves, areas with low and high levels of activatable NPF correspond to the inner and outer areas, respectively. Similar differences in molecular composition between the inner area, enriched by $\mathrm{PIP}_{3}$ and Ras activities, and the outer area, enriched by 25 PTEN, cortexillin, and myosin II, has been observed in experiments. Future work is needed to understand 26 the causes and implications of these localized activities.

${ }_{27}$ In the current model, all filaments remain orthogonal to the membrane until they disappear due to 28 depolymerization. Thus the activation and diffusion of barbed-end-activated signal (here it is NPF), 29 coupled with a positive feedback, leads to actin wave propagation in the model. This is in contrast 30 with other models described earlier wherein filament orientation plays a significant role. It's not known 
1 whether propagation depends to an extent on formation of filaments parallel to the membrane, but it is

2 likely that both mechanisms contribute to the wave formation and propagation. For a system without

3 any pre-existing actin filaments, the wave precursor - an actin spot - is a consequence of the combined

4 actions of local NPF activation and spontaneous filament nucleation and both are indispensable. The

5 fact that all filaments are orthogonal is not a restrictive assumption here, since forces are not taken into 6 account.

The current work incorporates a link between the actin assembly machinery and adhesion activity at 8 the plasma membrane. However, there is only one molecular player mediating the actin polymerization 9 and possible membrane activity, namely NPF. In vivo, a complicated signaling cascade involving the 10 integrin/PI3K/Rac pathway may be involved in the actin wave dynamics [27,40], and these signaling 11 components can be integrated into the stochastic model as more details become known. In the current 12 model waves can only propagate outward, but the experimentally-observed standing waves that reverse 13 direction could be a consequence of two competing F-actin upstream signals, namely PI3K and PTEN. 14 It is believed that PI3K stimulates $\mathrm{PIP}_{3}$ production and thus leads to F-actin polymerization, whereas 15 PTEN converts $\mathrm{PIP}_{3}$ to $\mathrm{PIP}_{2}$ and thus inhibits actin polymerization. The PTEN intrusion into an F-actin 16 wave has proven able to break waves and alter their direction of travel [36]. Moreover, the competition 17 between PI3K and PTEN activities may explain the experimentally-observed sharp transition in the ${ }_{18} \mathrm{PIP}_{3}$ level at the peak of actin waves. The possibility of explaining the reversal of propagating waves by 19 incorporating this signaling cascade will be explored in the future.

\section{${ }_{20}$ Materials and Methods}

\section{${ }_{21}$ Equation systems}

22 The system domain is the rectangular solid $\Omega^{3 d}=\left[0, L_{x}\right] \times\left[0, L_{y}\right] \times\left[0, L_{z}\right]$, where $L_{x}, L_{y}, L_{z}$ are the lengths in the three axial directions. The interior of $\Omega^{3 d}$ represents the cytosol, and the membrane is ${ }_{24}$ represented by the plane $\Omega^{2 d}=\left[0, L_{x}\right] \times\left[0, L_{y}\right] \times[z=0]$. The state variables are divided into three 25 groups: the diffusible species in the cytosol, membrane-bound species and filament-associated species.

${ }_{26}$ We suppress the presence of time and space variables in equations for the evolution of the state variables ${ }_{27}$ unless they are needed for clarity. The definitions and values of the parameters used in the equations are 28 defined in the next section. 
1 The evolution of the mobile cytosolic species - G-actin (g), Arp2/3 (arp), coronin (cor)and CP (cp) 2 proteins - are governed by

$$
\begin{aligned}
\frac{\partial[g]}{\partial t} & =D_{g} \nabla^{2}[g]+\mathcal{R}_{g} \\
\frac{\partial[\operatorname{arp}]}{\partial t} & =D_{\text {arp }} \nabla^{2}[\text { arp }]+\mathcal{R}_{p 1} \\
\frac{\partial[c p]}{\partial t} & =D_{c p} \nabla^{2}[c p]+\mathcal{R}_{c p} \\
\frac{\partial[\text { cor }]}{\partial t} & =D_{c o r} \nabla^{2}[\text { cor }]-\mathcal{R}_{p 2}+\mathcal{R}_{p 1}
\end{aligned}
$$

7 with reflective boundary conditions on the surface $\partial \Omega^{3 d}$ except on the membrane $\Omega^{2 d}$, and there

$$
\begin{aligned}
& -\left.D_{g} \frac{\partial}{\partial z}[g]\right|_{z=0}=-\left.k_{b k}^{+}[g]\right|_{z=0} \cdot F_{b k f r e e} \\
& -\left.k_{\text {gan }}^{+}[g]\right|_{z=0} \cdot\left[n p f^{*} \_a r p\right]+k_{g a n}^{-}\left[n p f^{*} \_a r p_{-} g\right] \\
& -\left.D_{\operatorname{arp}} \frac{\partial}{\partial z}[\operatorname{arp}]\right|_{z=0}=-\left.k_{a n}^{+}[\operatorname{arp}]\right|_{z=0} \cdot\left[n p f^{*}\right]+k_{\text {an }}^{-}\left[n p f^{*} \_a r p\right] \\
& -\left.D_{c p} \frac{\partial}{\partial z}[c p]\right|_{z=0}=-\left.k_{c a p}^{+}[c p]\right|_{z=0} \cdot F_{b k f r e e} \\
& -\left.D_{\text {cor }} \frac{\partial}{\partial z}[\operatorname{cor}]\right|_{z=0}=0
\end{aligned}
$$

13 where $\mathcal{R}$ 's represent various reactions at filament ends, and $F_{b k f r e e}$ the concentration of backbone fila- 
1 ments

$$
\begin{aligned}
& \mathcal{R}_{g}=\frac{1}{h_{i+1}-h_{i}} \sum_{\frac{2 h_{i}}{\delta} \leq n<\frac{2 h_{i+1}}{\delta}}\left\{k_{p k}^{-} f_{k}(n)+k_{p r}^{-} \sum_{n_{b} \leq n-2, b_{\text {tag }}} f_{r}\left(n_{b}, n-n_{b}, b_{t a g}, 0\right)\right. \\
& \left.-\left.k_{b r}^{+}[g]\right|_{z=0} \sum_{n_{L}, p_{\text {tag }}} f_{r}\left(n, n_{L}, 0, p_{\text {tag }}\right)\right\} \\
& \mathcal{R}_{c p}=\frac{1}{h_{i+1}-h_{i}} \sum_{\frac{2 h_{i}}{\delta} \leq n<\frac{2 h_{i+1}}{\delta}}\left\{k_{p r}^{-} f_{r}(n-1,2,1,0)\right. \\
& \left.-k_{\text {cap }}^{+}[c a p] \sum_{n_{L}, p_{\text {tag }}} f_{r}\left(n, n_{L}, 0, p_{\text {tag }}\right)\right\} \\
& \mathcal{R}_{p 1}=\frac{k_{\text {arp_cor }}^{-}}{h_{i+1}-h_{i}} \sum_{\frac{2 h_{i}}{\delta} \leq n<\frac{2 h_{i+1}}{\delta}}\left\{\sum_{n_{b} \leq n-2, b_{\text {tag }}} f_{r}\left(n_{b}, n-n_{b}, b_{\text {tag }}, 1\right)\right\} \\
& \mathcal{R}_{p 2}=\frac{k_{\text {arp_cor }}^{+}[\mathrm{cor}]}{h_{i+1}-h_{i}} \sum_{\frac{2 h_{i}}{\delta} \leq n<\frac{2 h_{i+1}}{\delta}}\left\{\sum_{n_{b} \leq n-2, b_{\text {tag }}} f_{r}\left(n_{b}, n-n_{b}, b_{\text {tag }}, 1\right)\right\} \\
& F_{\text {bkfree }}=\sum_{n_{L}} f_{k}\left(n_{L}\right)
\end{aligned}
$$

10 where the $f_{k}$ 's are the concentrations of backbone filaments consisting of $n$ monomers, and $h_{i+1}$ and $h_{i}$ are

11 the z-position of lower and upper surfaces of the $i$-th discretization in the z-direction for cytosolic species,

12 respectively. Similarly $f_{r}\left(n_{b}, n_{L}, b_{t a g}, p_{t a g}\right)$ is the concentration of branched filaments of length $n_{L}$ with

13 barbed end positioned at $n_{b}$, which is $n_{b}$-monomers away from the membrane. $b_{\text {tag }}(=0,1)$ indicates the

14 capping state of barbed end (free and capped, respectively), whereas $p_{\text {tag }}(=0,1,2)$ indicates the pointed

15 end state - either free, Arp2/3-capped or Arp2/3-coronin-capped.

${ }_{16}$ The proteins that reside on the membrane are the various states of NPF's and their association

17 with Arp2/3 and G-actin. We allow 2D diffusion for free (non-complexed) states of NPF's, but not for 
1 complexes. The dynamics of these state variables satisfy

$$
\begin{aligned}
& \frac{\partial[n p f]}{\partial t}=D_{n p f} \nabla^{2}[n p f]-k_{a c t v} F_{b r f r e e} \cdot[n p f]+k_{d e g}\left[n p f^{*}\right]+k_{\text {recov }}\left[n p f^{* *}\right] \\
& \frac{\partial\left[n p f^{*}\right]}{\partial t}=D_{n p f^{*}} \nabla^{2}\left[n p f^{*}\right]+k_{a c t v} F_{b r f r e e} \cdot[n p f] \\
& -k_{d e g}\left[n p f^{*}\right]-\left.k_{a n}^{+}[\operatorname{arp}]\right|_{z=0} \cdot\left[n p f^{*}\right]+k_{a n}^{-}\left[n p f^{*}{ }_{-} a r p\right] \\
& \frac{\partial\left[n p f^{*}{ }_{\_} a r p\right]}{\partial t}=\left.k_{a n}^{+}[\operatorname{arp}]\right|_{z=0} \cdot\left[n p f^{*}\right]-k_{a n}^{-}\left[n p f^{*}{ }_{\text {_arp }}\right] \\
& -\left.k_{\text {gan }}^{+}[g]\right|_{z=0} \cdot\left[n p f^{*} \_a r p\right]+k_{\text {gan }}^{-}\left[n p f^{*}{ }_{\_} a r p_{-} g\right] \\
& \frac{\partial\left[n p f^{*}{ }_{\_} a r p_{-} g\right]}{\partial t}=\left.k_{g a n}^{+}[g]\right|_{z=0} \cdot\left[n p f^{*}{ }_{\_} a r p\right]-k_{g a n}^{-}\left[n p f^{*}{ }_{-} a r p_{-} g\right]-k_{n u c l}\left[n p f^{*}{ }_{\_} a r p_{-} g\right] \cdot F_{b t o t} \\
& \frac{\partial\left[n p f^{* *}\right]}{\partial t}=D_{n p f^{* *}} \nabla^{2}\left[n p f^{* *}\right]+k_{n u c l}\left[n p f^{*}{ }_{-a r p} g\right] \cdot F_{b t o t}-k_{\text {recov }}\left[n p f^{* *}\right]
\end{aligned}
$$

9 on the domain $\Omega^{2 d}$, with reflective boundary conditions at $\partial \Omega^{2 d}$. The averaged concentrations of free

10 barbed ends and total barbed ends of branched filaments within the nucleation zone adjacent to the

11 membrane are

$$
\begin{aligned}
F_{\text {brfree }} & =\frac{1}{L_{\text {nucl_zone }}} \sum_{n \leq \frac{2 L_{n u c l \_z o n e}}{\delta}}\left\{\sum_{n_{L}, p_{\text {tag }}} f_{r}\left(n, n_{L}, 0, p_{\text {tag }}\right)\right\} \\
F_{\text {btot }} & =\frac{1}{L_{\text {nucl_zone }}}\left\{\sum_{n_{L}} f_{k}\left(n_{L}\right)+\sum_{n \leq \frac{2 L_{\text {nucl_zone }}}{\delta}}\left(\sum_{n_{L}, b_{\text {tag }}, p_{\text {tag }}} f_{r}\left(n, n_{L}, b_{\text {tag }}, p_{\text {tag }}\right)\right)\right\} .
\end{aligned}
$$

Backbone filaments are generated on nucleation sites and remain attached to the sites until it is 15 capped and thus considered as a member of the connected branched filaments. We assume the latter 16 as a rigid filament cluster, which is able to move vertically due to the polymerization at the membrane17 adjacent barbed end of any member filament. The nucleation site is occupied by attached backbone filament and cannot nucleate new backbone filament until the occupied one is capped. Note there is not lateral movement of nucleation sites and filaments on the membrane in this model. Let $S_{f}$ denote the 
1 concentration of free nucleation sites for backbone filament. The dynamics of these species satisfy

$$
\begin{aligned}
\frac{\partial S_{f}}{\partial t}= & -\left.k_{n u k}^{+}[g]\right|_{z=0} S_{f}+\left.k_{c a p}^{+}[c p]\right|_{z=0} \sum_{n \geq 2} f_{k}(n)+k_{n u k}^{-} f_{k}(1) \\
\frac{\partial f_{k}(1)}{\partial t}= & \left.k_{n u k}^{+}[g]\right|_{z=0} S_{f}-k_{n u k}^{-} f_{k}(1)+k_{p k}^{-} f_{k}(2)-\left.k_{b k}^{+}[g]\right|_{z=0} f_{k}(1) \\
\frac{\partial f_{k}(n)}{\partial t}= & \left.k_{b k}^{+}[g]\right|_{z=0} f_{k}(n-1)+k_{p k}^{-} f_{k}(n+1) \\
& -\left(\left.k_{b k}^{+}[g]\right|_{z=0}+k_{p k}^{-}\right) f_{k}(n)-\left.k_{c a p}^{+}[c p]\right|_{z=0} f_{k}(n), \quad(n \geq 2)
\end{aligned}
$$

6 The dynamics of the branched filament is dictated by the filament-end reactions, which include the 7 Arp2/3 removal facilitated by coronin binding and subsequent depolymerization at the pointed end, and 8 polymerization and capping at the barbed end. The detailed evolution follows as

$$
\begin{aligned}
& \frac{\partial f_{r}(1,1,0,1)}{\partial t}=k_{n u r}^{+}\left[g_{-} a r p \_n p f^{*}\right]\left\{\sum_{n \geq 1} f_{k}(n)+\sum_{b_{\text {tag }}, n, p_{\text {tag }}} f_{r}\left(1, n, b_{\text {tag }}, p_{\text {tag }}\right)\right\} \\
& -\left.k_{\text {arp_cor }}^{+}[c o r]\right|_{z=\delta} f_{r}(1,1,0,1)-v_{b} \cdot f_{r}(1,1,0,1) \\
& \frac{\partial f_{r}(i, 1,0,1)}{\partial t}=k_{n u r}^{+}\left[g_{-} a r p \_n p f^{*}\right] \sum_{b_{\text {tag }}, n, p_{\text {tag }}} f_{r}\left(i, n, b_{\text {tag }}, p_{\text {tag }}\right) \\
& -\left.k_{b r}^{+}[g]\right|_{z=\frac{i \delta}{2}} f_{r}(i, 1,0,1)-\left.k_{a r p_{-} c o r}^{+}[\operatorname{cor}]\right|_{z=\frac{(i+1) \delta}{2}} f_{r}(i, 1,0,1) \\
& -v_{b} \cdot\left\{f_{r}(i, 1,0,1)-f_{r}(i-1,1,0,1)\right\}, \quad 1<i \leq \frac{2 L_{\text {nucl_zone }}}{\delta} \\
& \frac{\partial f_{r}(j, 1,0,1)}{\partial t}=-\left.k_{b r}^{+}[g]\right|_{z=\frac{j \delta}{2}} f_{r}(j, 1,0,1)-\left.k_{\text {arp_cor }}^{+}[\operatorname{cor}]\right|_{z=\frac{(j+1) \delta}{2}} f_{r}(j, 1,0,1) \\
& -v_{b} \cdot\left\{f_{r}(j, 1,0,1)-f_{r}(j-1,1,0,1)\right\}, \quad j>\frac{2 L_{\text {nucl_zone }}}{\delta} \\
& \frac{\partial f_{r}(1,1,0,2)}{\partial t}=\left.k_{a r p_{-} c o r}^{+}[\operatorname{cor}]\right|_{z=\delta} f_{r}(1,1,0,1) \\
& -v_{b} \cdot f_{r}(1,1,0,2)-k_{\text {arp_cor }}^{-} f_{r}(1,1,0,2) \\
& \frac{\partial f_{r}(i, 1,0,2)}{\partial t}=-\left.k_{b r}^{+}[g]\right|_{z=\frac{i \delta}{2}} f_{r}(i, 1,0,2)+\left.k_{a r p \_c o r}^{+}[\operatorname{cor}]\right|_{z=\frac{(i+1) \delta}{2}} f_{r}(i, 1,0,1) \\
& -k_{\text {arp_cor }}^{-} f_{r}(i, 1,0,2) \\
& -v_{b} \cdot\left\{f_{r}(i, 1,0,2)-f_{r}(i-1,1,0,2)\right\}, \quad i>1
\end{aligned}
$$


where

$$
v_{b}=\left.k_{b r}^{+}[g]\right|_{z=\frac{\delta}{2}} \cdot \chi\left(\sum_{n_{L}, p_{\text {tag }}} f_{r}\left(1, n_{L}, 0, p_{\text {tag }}\right)\right)
$$

and

$$
\chi(n)= \begin{cases}1, & n>0 \\ 0, & n=0\end{cases}
$$

For filaments whose lengths $n_{L} \geq 2$, one has

$$
\begin{aligned}
& \frac{\partial f_{r}\left(1, n_{L}, 0,1\right)}{\partial t}=\left.k_{b r}^{+}[g]\right|_{z=\frac{\delta}{2}} f_{r}\left(1, n_{L}-1,0,1\right)+\left.k_{b r}^{+}[g]\right|_{z=\delta} f_{r}\left(2, n_{L}-1,0,1\right) \\
& -v_{b} \cdot f_{r}\left(1, n_{L}, 0,1\right) \\
& -\left\{k_{\text {arp_cor }}^{+}[\mathrm{cor}]+k_{\text {cap }}^{+}[\mathrm{cap}]\right\} f_{r}\left(1, n_{L}, 0,1\right) \\
& \frac{\partial f_{r}\left(i, n_{L}, 0,1\right)}{\partial t}=\left.k_{b r}^{+}[g]\right|_{z=\frac{(i+1) \delta}{2}} f_{r}\left(i+1, n_{L}-1,0,1\right)-\left.k_{b r}^{+}[g]\right|_{z=\frac{i \delta}{2}} f_{r}\left(i, n_{L}, 0,1\right) \\
& -v_{b} \cdot\left\{f_{r}\left(i, n_{L}, 0,1\right)-f_{r}\left(i-1, n_{L}, 0,1\right)\right\} \\
& -\left\{k_{\text {arp_cor }}^{+}[\mathrm{cor}]+k_{\text {cap }}^{+}[\mathrm{cap}]\right\} f_{r}\left(i, n_{L}, 0,1\right), \quad i>1 \\
& \frac{\partial f_{r}\left(1, n_{L}, 0,2\right)}{\partial t}=\left.k_{b r}^{+}[g]\right|_{z=\frac{\delta}{2}} f_{r}\left(1, n_{L}-1,0,2\right)+\left.k_{b r}^{+}[g]\right|_{z=\delta} f_{r}\left(2, n_{L}-1,0,2\right) \\
& -v_{b} \cdot f_{r}\left(1, n_{L}, 0,2\right) \\
& +\left.k_{\text {arp_cor }}^{+}[\text {cor }]\right|_{z=\frac{\left(n_{L}+1\right) \delta}{2}} f_{r}\left(1, n_{L}, 0,1\right) \\
& -\left.k_{\text {cap }}^{+}[c a p]\right|_{z=\frac{\delta}{2}} f_{r}\left(1, n_{L}, 0,2\right)-k_{\text {arp_cor }}^{-} f_{r}\left(1, n_{L}, 0,2\right) \\
& \frac{\partial f_{r}\left(i, n_{L}, 0,2\right)}{\partial t}=\left.k_{b r}^{+}[g]\right|_{z=\frac{(i+1) \delta}{2}} f_{r}\left(i+1, n_{L}-1,0,2\right)-\left.k_{b r}^{+}[g]\right|_{z=\frac{i \delta}{2}} f_{r}\left(i, n_{L}, 0,2\right) \\
& -v_{b} \cdot\left\{f_{r}\left(i, n_{L}, 0,2\right)-f_{r}\left(i-1, n_{L}, 0,2\right)\right\} \\
& +\left.k_{\text {arp_cor }}^{+}[\mathrm{cor}]\right|_{z=\frac{\left(n_{L}+i\right) \delta}{2}} f_{r}\left(i, n_{L}, 0,1\right) \\
& -\left.k_{\text {cap }}^{+}[\operatorname{cap}]\right|_{z=\frac{i \delta}{2}} f_{r}\left(i, n_{L}, 0,2\right)-k_{\text {arp_cor }}^{-} f_{r}\left(i, n_{L}, 0,2\right), \quad i>1
\end{aligned}
$$




$$
\begin{aligned}
& \frac{\partial f_{r}\left(1, n_{L}, 0,0\right)}{\partial t}=\left.\quad k_{b r}^{+}[g]\right|_{z=\frac{\delta}{2}} f_{r}\left(1, n_{L}-1,0,0\right)+\left.k_{b r}^{+}[g]\right|_{z=\delta} f_{r}\left(2, n_{L}-1,0,0\right) \\
&-v_{b} \cdot f_{r}\left(1, n_{L}, 0,2\right) \\
&-k_{p r}^{-}\left\{f_{r}\left(1, n_{L}, 0,0\right)-f_{r}\left(1, n_{L}+1,0,0\right)\right\} \\
& \frac{\partial f_{r}\left(i, n_{L}, 0,0\right)}{\partial t}=\left.k_{b r}^{+}[g]\right|_{z=\frac{(i+1) \delta}{2}} f_{r}\left(i+1, n_{L}-1,0,0\right)-\left.k_{b r}^{+}[g]\right|_{z=\frac{i \delta}{2}} f_{r}\left(i, n_{L}, 0,0\right) \\
&-k_{p r}^{-}\left\{f_{r}\left(i, n_{L}, 0,0\right)-f_{r}\left(i, n_{L}+1,0,0\right)\right\} \\
&-v_{b} \cdot\left\{f_{r}\left(i, n_{L}, 0,0\right)-f_{r}\left(i-1, n_{L}, 0,0\right)\right\} \\
&-\left.k_{c a p}^{+}[\operatorname{cap}]\right|_{z=\frac{i \delta}{2}} f_{r}\left(i, n_{L}, 0,0\right)+k_{\text {arp } \_ \text {cor }}^{-} f_{r}\left(i, n_{L}, 0,2\right), \quad i>1
\end{aligned}
$$

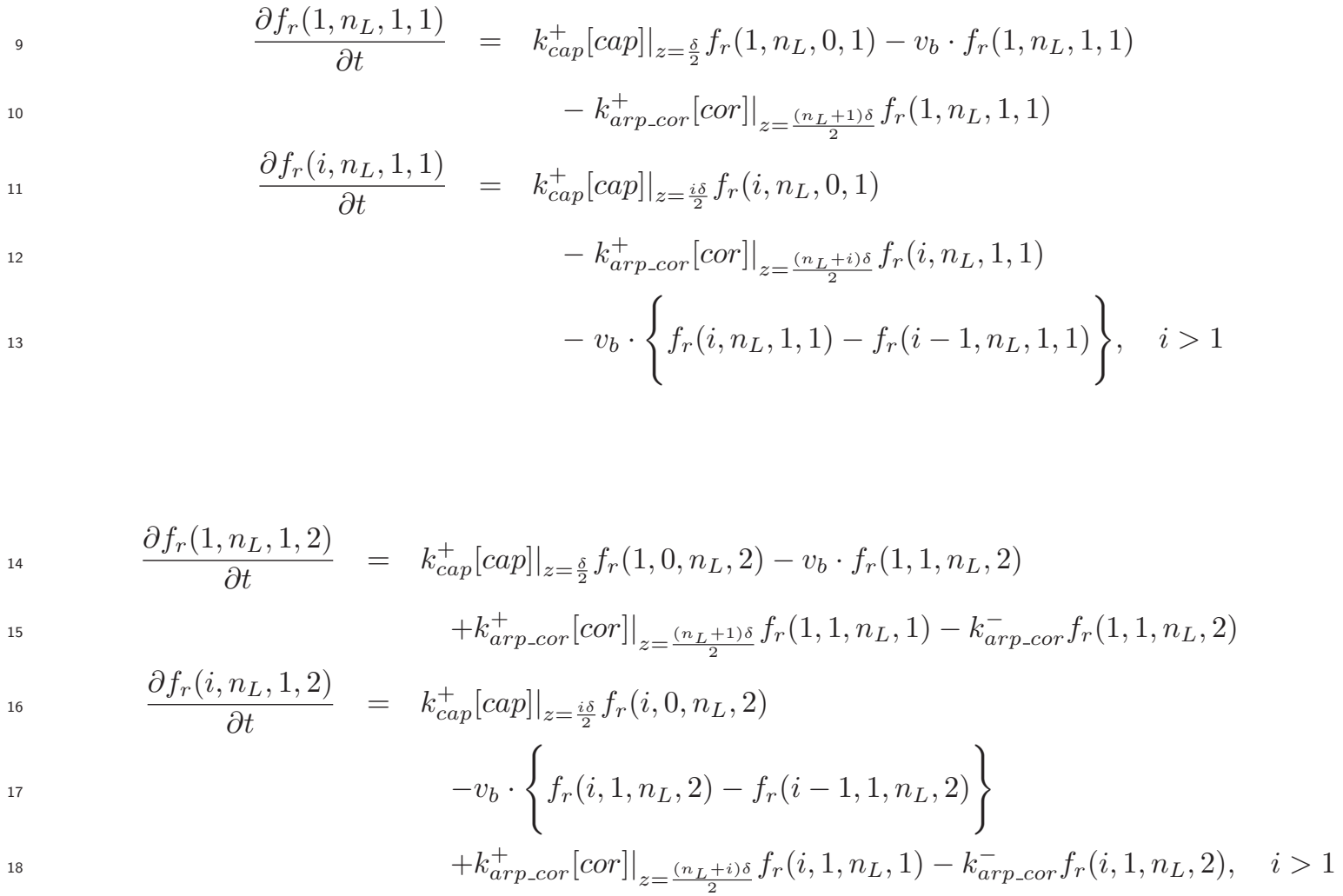




$$
\begin{aligned}
\frac{\partial f_{r}\left(1, n_{L}, 1,0\right)}{\partial t}= & \left.k_{\text {cap }}^{+}[\operatorname{cap}]\right|_{z=\frac{\delta}{2}} f_{r}\left(1, n_{L}, 0,0\right)-v_{b} \cdot f_{r}\left(1, n_{L}, 1,0\right) \\
& +k_{p r}^{-}\left\{f_{r}\left(1, n_{L}+1,1,0\right)-f_{r}\left(1, n_{L}, 1,0\right)\right\} \\
& +k_{\text {arp_cor }}^{-} f_{r}\left(1, n_{L}, 1,2\right)+\left.k_{\text {cap }}^{+}[\operatorname{cap}]\right|_{z=\frac{\delta}{2}} f_{k}\left(n_{L}\right) \\
\frac{\partial f_{r}\left(i, n_{L}, 1,0\right)}{\partial t}=\left.\quad k_{c a p}^{+}[\operatorname{cap}]\right|_{z=\frac{i \delta}{2}} f_{r}\left(i, n_{L}, 0,0\right) & \\
& -v_{b} \cdot\left\{f_{r}\left(i, n_{L}, 1,0\right)-f_{r}\left(i-1, n_{L}, 1,0\right)\right\} \\
& +k_{p r}^{-}\left\{f_{r}\left(i, n_{L}+1,1,0\right)-f_{r}\left(i, 1, n_{L}, 1,0\right)\right\} \\
& +k_{\text {arp_cor }}^{-} f_{r}\left(i, n_{L}, 1,2\right), \quad i>1
\end{aligned}
$$

\section{\& Parameters}

9 We use the following benchmark set of parameters for all stochastic simulations of the model, except for cases where selected particular parameters are changed to examine the resulting effect of those parameters.

11 The parameters which are not referenced are chosen to produce experimentally-compatible wave behavior.

12 Referenced parameters are chosen either the same as or within the normal range as in the literature.

There exist three major differences between the parameters used here and those in literature. Firstly, the depolymerization rate at filament pointed ends is 8-10 times faster than the depolymerization of pure filaments. The faster rate reflects the combined effect of Aip1, cofilin and coronin, which contribute to the filament destabilization and rapid pointed-end depolymerization [41]. Second is the diffusion rate constant of membrane-bound molecules such as various NPF proteins, which is ten-fold slow than those membrane-bound and free diffusing molecules used in other studies [34]. The values used are at the lower end of the range of values for diffusion of proteins in a membrane, but reflect the fact that these are effective rates that incorporate binding to scaffolding proteins, etc. The third group is the kinetic rate constants involving the filament branching. Here the rate constants are higher, which could be justified

22 provided that most filament branching occurs at the cytoskeleton-membrane interface. The rate constants

23 suggested in experiments are usually derived from bulk reactions in 3D solution [42]. 
Table 1. Parameters used in the actin wave model

\begin{tabular}{|c|c|c|c|}
\hline Symbol & Meaning & Value & Reference \\
\hline$D_{g}$ & diffusion constant of G-actin in cytosol & $4.0 \mu \mathrm{m}^{2} / \mathrm{s}$ & {$[34]$} \\
\hline$D_{a r p}$ & diffusion constant of Arp $2 / 3$ in cytosol & $4.0 \mu \mathrm{m}^{2} / \mathrm{s}$ & {$[34]$} \\
\hline$D_{\text {cor }}$ & diffusion constant of coronin in cytosol & $4.0 \mu \mathrm{m}^{2} / \mathrm{s}$ & {$[34]$} \\
\hline$D_{c p}$ & diffusion constant of $\mathrm{CP}$ in cytosol & $4.0 \mu \mathrm{m}^{2} / \mathrm{s}$ & {$[34]$} \\
\hline$D_{n p f}$ & diffusion constant of NPF on membrane & $0.005 \mu \mathrm{m}^{2} / \mathrm{s}$ & \\
\hline$D_{n p f^{*}}$ & diffusion constant of $\mathrm{NPF}^{*}$ on membrane & $0.005 \mu \mathrm{m}^{2} / \mathrm{s}$ & \\
\hline$D_{n p f^{* *}}$ & diffusion constant of $\mathrm{NPF}^{* *}$ on membrane & $0.005 \mu \mathrm{m}^{2} / \mathrm{s}$ & \\
\hline$k_{b r}^{+}$ & on-rate of monomer at branched barbed ends & $10.0 \mu M^{-1} s^{-1}$ & {$[43]$} \\
\hline$k_{p r}^{-}$ & off-rate of monomer at branched pointed ends & $100.0 s^{-1}$ & \\
\hline$k_{b k}^{+}$ & on-rate of monomer at backbone barbed ends & $10.0 \mu M^{-1} s^{-1}$ & {$[43]$} \\
\hline$k_{p k}^{-n}$ & off-rate of monomer at backbone pointed ends & $100.0 s^{-1}$ & \\
\hline$k_{n u k}^{+}$ & on-rate of backbone filament nucleation & $10.0 \mu M^{-1} s^{-1}$ & {$[43]$} \\
\hline$k_{n u k}^{-}$ & off-rate of backbone filament nucleation & $1000.0 s^{-1}$ & {$[43]$} \\
\hline$k_{c a p}^{+}$ & on-rate of $\mathrm{CP}$ at filament barbed end & $3.0 \mu M^{-1} s^{-1}$ & {$[44]$} \\
\hline$k_{\text {arp_cor }}^{+}$ & on-rate of coronin binding to Arp $2 / 3$ & $3.0 \mu M^{-1} s^{-1}$ & \\
\hline$k_{\text {arp_cor }}^{-}$ & off-rate of coronin-Arp2/3 complex & $0.5 s^{-1}$ & \\
\hline$k_{a c t v}$ & activation rate of NPF by free barbed ends & $5.0 \mu M^{-1} s^{-1}$ & \\
\hline$k_{\text {deg }}$ & decay rate of $\mathrm{NPF}$ & $0.1 s^{-1}$ & \\
\hline$k_{\text {recov }}$ & recovery rate of $\mathrm{NPF}$ & $0.01 s^{-1}$ & \\
\hline$k_{a n}^{+}$ & on-rate of Arp2/3 bind to active $\mathrm{NPF}$ & $10.0 \mu M^{-1} s^{-1}$ & \\
\hline$k_{a n}^{-}$ & off-rate of Arp2/3 bind to active NPF & $1.0 \mathrm{~s}^{-1}$ & \\
\hline$k_{\text {gan }}^{+}$ & on-rate of G-actin bind to NPF-Arp2/3 complex & $10.0 \mu M^{-1} s^{-1}$ & \\
\hline$k_{\text {gan }}^{-}$ & off-rate of G-actin bind to NPF-Arp2/3 complex & $1.0 s^{-1}$ & \\
\hline$k_{\text {nur }}^{+}$ & nucleation rate of branched filaments & $5.0 \mu M^{-1} s^{-1}$ & \\
\hline
\end{tabular}

\section{Simulation algorithm and numerical method}

2 The membrane domain is partitioned into square compartments of size $l_{x} \times l_{y}$, and the cytoplasmic space

3 into cubic compartments of size $l_{x} \times l_{y} \times l_{z}$, where the side lengths are all $0.1 \mu m$. This is small enough

4 that each compartment can be considered well-mixed. The Monte Carlo method is used to generate

5 realizations of the stochastic model, and specifically, we implement the numerical algorithm using a

6 modified Gillespie direct method developed by Matzavinos and Othmer [46] (MO hereafter). In the

7 original Gillespie direct method, two random numbers are generated for advancing the model system in

8 each time step: one random number is used to determine the waiting time for the next reaction, and the 
Table 2. Parameters applied in the actin wave model (continued)

\begin{tabular}{|c|c|c|c|}
\hline Symbol & Meaning & Value & Reference \\
\hline$L_{x}$ & system size in $\mathrm{X}$-direction & $5.0 \mu \mathrm{m}$ & \\
\hline$L_{y}$ & system size in Y-direction & $5.0 \mu \mathrm{m}$ & \\
\hline$L_{z}$ & system size in Z-direction & $2.0 \mu \mathrm{m}$ & \\
\hline$l_{x}$ & compartment size in $\mathrm{X}$-direction & $0.1 \mu m$ & \\
\hline$l_{y}$ & compartment size in Y-direction & $0.1 \mu m$ & \\
\hline$l_{z}$ & compartment size in Z-direction & $0.1 \mu \mathrm{m}$ & \\
\hline$c_{m o n}$ & initial G-actin concentration & $10.0 \mu M$ & \\
\hline$c_{a r p 2 / 3}$ & initial Arp2/3 concentration & $1.0 \mu M$ & {$[35]$} \\
\hline$c_{\text {coronin }}$ & initial coronin concentration & $1.0 \mu M$ & {$[35]$} \\
\hline$c_{c p}$ & initial CP concentration & $1.0 \mu M$ & {$[35]$} \\
\hline$c_{n p f}$ & initial NPF concentration & $3000 / \mu m^{2}$ & {$[45]$} \\
\hline$c_{n u c l}$ & surface density of backbone filament nucleation site & $100 / \mu m^{2}$ & {$[39]$} \\
\hline$L_{\text {nucl_zone }}$ & $\begin{array}{l}\text { maximal distant where free barbed ends can } \\
\text { activate NPF and formed new filament branches }\end{array}$ & $0.0135 \mu \mathrm{m}$ & \\
\hline
\end{tabular}

1 other is used to determine which reaction type occurs [47]. In this method the reactions are distinguished

2 by the reactants involved, and therefore, for instance, the reaction of monomer depolymerization from

3 the pointed end of a filament of length $n$ is considered different from that of size $n+1$. In the MO

4 method, the state of the systems consists of equivalence classes of filaments characterized firstly by their

5 length, and then subdivided into classes of the same nucleotide profile. In the model developed here the

6 nucleotide profiles play no role. Then monomer depolymerization from filaments of any size is considered

7 as one reaction type in an equivalence class of reactants. Another reaction type consists of all the

8 reactions involving monomer addition at a barbed end, irrespective of how long the elongating filament

9 is, which is legitimate since the on-rate for monomer addition is independent of the filament length. Thus

10 a third random number is needed after the reaction type that occurs is determined in order to decide

11 which reaction within the equivalence class occurs. This treatment reduces the computational cost by

12 2-3 orders of magnitude by making an optimal use of the structure of underlying reaction network [46] .

${ }_{13}$ In the current discretization of the simulation domain, there are $N_{\text {cmprt }}=\left(L_{x} / l_{x} \times L_{y} / l_{y} \times L_{z} / l_{z}\right)$

14 compartments in the cytoplasmic domain, which is around 50,000 in typical computations. Each of

15 the $N_{\text {cmprt }}$ computational compartments is considered to be well-mixed, and there are pseudo-reactions

16 corresponding to diffusive hops between compartments. We lump the equivalence class of reactants of 
1 the same type in individual compartments into a large equivalence class in the whole domain. Following 2 the MO method, we first determine the waiting time for the next reaction from the prospensity of all 3 the allowable reactions, then decide which equivalent class of reactions over the whole domain occurs, 4 after which we decide which compartment this equivalent class belongs to. A direct search for which compartment the next reaction occurs in consumes a great deal of time to find the target compartment 6 by checking each compartment. We thus developed a search method by subdividing the total $N_{\text {cmprt }}$ compartments into $N_{\text {sub }}$ subsets. Instead of searching directly for the target compartment for the next reaction, we first search for which subset the target compartment belongs to, then determine the target compartment within that subset. We found that an optimal subdivision of the compartments can reduce the search time by a factor of $5-10$.

The detailed algorithm is as follows. Suppose that the system has $N_{\text {rct_type }}$ equivalent reaction classes and that the rate constant of the $\mathrm{j}$-th reaction type is $r_{j}$. Consider there are $N_{c m p r t}$ computational comparments, in the i-th of which there are $R A_{i}^{j}$ possible reactions for reaction type $\mathrm{j}$. Therefore, for the $\mathrm{j}$-th equivalent reaction class of the domain, we have total number of this reaction in the whole domain as $R A_{\text {tot }}^{j}=\sum_{i=1}^{N_{c m p r t}} R A_{i}^{j}$. In addition, suppose $\widehat{R A}_{k}^{j}$ denotes the total number of reactions of type $\mathrm{j}$ in the k-th subset in the totality of $N_{s u b}$ subsets. After setting the above system configuration, the state of the system is advanced as follows. At time $t_{i}$, the steps proceed as follows

1. Generate a random number to determine the waiting time $\Delta t_{i}$ for next reaction by the reaction prospensities derived from RAtot $^{j}$ and $r_{j}$ according to Gillespie direct method;

2. Generate a second random number, and decide which reaction type the next reaction will be from RAtot $^{j}$ and $r_{j}$ according to Gillespie direct method;

3. Generate a third random number and decide which reacting compartment the reaction type decided in Step 2 locates in. In this step, instead of checking the $N_{\text {cmprt }}$ compartment one by one, we first subdivide the compartments into subsets, determine in which subset the reacting compartment falls, and then within that subset determine the appropriate reaction compartment. In essence this is done as in step one, except that we compute total propensities within subsets and use these to determine the subset, in effect treating subsets as individual steps. (An optimization of the choice of the number of subsets is shown later.)

4. In the chosen compartment, we proceed as follows. 
- if the reactants for the chosen reaction are identical molecular species, pick any reactants to react. For example, for molecular diffusion, which molecule of the same type diffuses out of the current compartment makes no difference, since the combinatorial coefficient used in computing propensities reflects the identity of the species.

- if the reactants are not identical molecular species, then generate another random number to decide which reactant or reactant pair to react. For example, if the pointed-end depolymerization is to occur in the reacting compartment, the filaments whose pointed end lies in the comparment may be of different lengths, and thus we must randomly choose one from these filaments.

\section{${ }_{14}$ Acknowledgments}

15 This research is supported by NSF Grant DMS 0817529 and the University of Minnesota Supercomputing

16 Institute.

\section{${ }_{17}$ References}

\section{${ }_{18}$ References}

1. Sheetz MP, Felsenfeld D, Galbraith CG, Choquet D (1999) Cell migration as a five-step cycle. Biochemical Society Symposia 65: 233-43.

2. Weiner OD, Marganski WA, Wu LF, Altschuler SJ, Kirschner MW (2007) An actin-based wave generator organizes cell motility. PLoS biology 5: e221.

3. Asano Y, Nagasaki A, Uyeda TQP (2008) Correlated waves of actin filaments and PIP3 in dictyostelium cells. Cell Motility and the Cytoskeleton 65: 923-934. 
4. Etienne-Manneville S (2004) Cdc42-the centre of polarity. J Cell Sci 117: 1291.

5. Sanz-Moreno V, Gadea G, Ahn J, Paterson H, Marra P, et al. (2008) Rac activation and inactivation control plasticity of tumor cell movement. Cell 135: 510-523.

6. Sanz-Moreno V, Marshall CJ (2010) The plasticity of cytoskeletal dynamics underlying neoplastic cell migration. Current Opinion in Cell Biology 22: 690-696.

7. Katoh K, Kano Y, Amano M, Onishi H, Kaibuchi K, et al. (2001) Rho-kinase-mediated contraction of isolated stress fibers. J of Cell Biology 153: 569-583.

8. Pollard TD, Blanchoin L, Mullins RD (2000) Molecular mechanisms controlling actin filament dynamics in nonmuscle cells. Annu Rev Biophys Biomol Struct 29: 545-76.

9. Bretschneider T, Anderson K, Ecke M, Müller-Taubenberger A, Schroth-Diez B, et al. (2009) The three-dimensional dynamics of actin waves, a model of cytoskeletal self-organization. Biophysical journal 96: 2888-2900.

10. Schroth-Diez B, Gerwig S, Ecke M, Hegerl R, Diez S, et al. (2009) Propagating waves separate two states of actin organization in living cells. HFSP Journal 3: 412-427.

11. Gerisch G (2010) Self-organizing actin waves that simulate phagocytic cup structures. PMC biophysics 3: 7 .

12. Dai J, Ting-Beall HP, Hochmuth RM, Sheetz MP, Titus MA (1999) Myosin I contributes to the generation of resting cortical tension. Biophys Jour 77: 1168-1176.

13. Cai L, Marshall TW, Uetrecht AC, Schafer DA, Bear JE (2007) Coronin 1B coordinates Arp2/3 complex and cofilin activities at the leading edge. Cell 128: 915-929.

14. Gerisch G, Bretschneider T, Müller-Taubenberger A, Simmeth E, Ecke M, et al. (2004) Mobile actin clusters and traveling waves in cells recovering from actin depolymerization. Biophysical journal 87: 3493-3503.

15. Vicker MG (2002) Eukaryotic cell locomotion depends on the propagation of self-organized reactiondiffusion waves and oscillations of actin filament assembly. Experimental cell research 275: 54-66. 
16. Gerisch G, Ecke M, Schroth-Diez B, Gerwig S, Engel U, et al. (2009) Self-organizing actin waves as planar phagocytic cup structures. Cell adhesion \& migration 3: 373.

17. Iijima M, Devreotes P (2002) Tumor suppressor PTEN mediates sensing of chemoattractant gradients. Cell 109: 599-610.

18. Billadeau DD (2008) PTEN gives neutrophils direction. Nature Immunology 9: 716-718.

19. Parent CA (2004) Making all the right moves: chemotaxis in neutrophils and Dictyostelium. Current Opinion in Cell Biology 16: 4-13.

20. Pramanik M, et al. (2009) PTEN is a mechanosensing signal transducer for myosin II localization in Dictyostelium cells. Genes to Cells 14: 821.

21. Bosgraaf L, van Haastert PJM (2006) The regulation of myosin II in dictyostelium. European Journal of Cell Biology 85: 969-979.

22. Andrew N, Insall RH (2007) Chemotaxis in shallow gradients is mediated independently of PtdIns 3-kinase by biased choices between random protrusions. Nature Cell Biology 9: 193-200.

23. King JS, Insall RH (2009) Chemotaxis: finding the way forward with Dictyostelium. Trends in Cell Biology 19: 523-530.

24. Soll DR, Wessels D, Kuhl S, Lusche DF (2009) How a cell crawls and the role of cortical myosin II. Eukaryotic Cell 8: 1381.

25. Pollitt AY, Insall RH (2009) WASP and SCAR/WAVE proteins: the drivers of actin assembly. Journal of cell science 122: 2575-2578.

26. Cai L, Makhov AM, Schafer DA, Bear JE (2008) Coronin 1B antagonizes cortactin and remodels arp2/3-containing actin branches in lamellipodia. Cell 134: 828-842.

27. Case LB, Waterman CM (2011) Adhesive F-actin waves: A novel integrin-mediated adhesion complex coupled to ventral actin polymerization. PLoS ONE 6: e26631.

28. Serrels B, Serrels A, Brunton VG, Holt M, McLean GW, et al. (2007) Focal adhesion kinase controls actin assembly via a FERM-mediated interaction with the arp2/3 complex. Nature cell biology 9 : $1046-1056$. 
29. Cornillon S, Gebbie L, Benghezal M, Nair P, Keller S, et al. (2006) An adhesion molecule in free-living dictyostelium amoebae with integrin $\beta$ features. EMBO reports 7: 617-621.

30. Schroth-Diez B, Gerwig S, Ecke M, Hegerl R, Diez S, et al. (2009) Propagating waves separate two states of actin organization in living cells. HFSP journal 3: 412-427.

31. Sasaki AT, Janetopoulos C, Lee S, Charest PG, Takeda K, et al. (2007) G protein-independent ras/PI3K/F-actin circuit regulates basic cell motility. The Journal of cell biology 178: 185 .

32. Beemiller P, Zhang Y, Mohan S, Levinsohn E, Gaeta I, et al. (2010) A cdc42 activation cycle coordinated by pi 3-kinase during fc receptor-mediated phagocytosis. Molecular biology of the cell 21: $470-480$.

33. Whitelam S, Bretschneider T, Burroughs NJ (2009) Transformation from spots to waves in a model of actin pattern formation. Physical review letters 102: 198103.

34. Carlsson AE (2010) Dendritic actin filament nucleation causes traveling waves and patches. Physical review letters 104: 228102.

35. Sackmann E, Keber F, Heinrich D (2010) Physics of cellular movements. Annual Review of Condensed Matter Physics .

36. Gerisch G, Ecke M, Wischnewski D, Schroth-Diez B (2011) Different modes of state transitions determine pattern in the phosphatidylinositide-actin system. BMC Cell Biology 12: 42.

37. Lai FPL, Szczodrak M, Block J, Faix J, Breitsprecher D, et al. (2008) Arp2/3 complex interactions and actin network turnover in lamellipodia. The EMBO journal 27: 982-992.

38. Millius A, Watanabe N, Weiner OD (2012) Diffusion, capture and recycling of SCAR/WAVE and arp2/3 complexes observed in cells by single-molecule imaging. Journal of Cell Science .

39. Chia CP, Shariff A, Savage SA, Luna EJ (1993) The integral membrane protein, ponticulin, acts as a monomer in nucleating actin assembly. The Journal of cell biology 120: 909-922.

40. DeMali KA, Barlow CA, Burridge K (2002) Recruitment of the arp2/3 complex to vinculin: coupling membrane protrusion to matrix adhesion. The Journal of cell biology 159: 881-891. 
41. Kueh HY, Charras GT, Mitchison TJ, Brieher WM (2008) Actin disassembly by cofilin, coronin, and aip1 occurs in bursts and is inhibited by barbed-end cappers. The Journal of cell biology 182: $341-353$.

42. Beltzner CC, Pollard TD (2008) Pathway of actin filament branch formation by arp2/3 complex. Journal of Biological Chemistry 283: 7135-7144.

43. Hu J, Matzavinos A, Othmer HG (2007) A theoretical approach to actin filament dynamics. Journal of Statistical Physics 128: 111-138.

44. Schafer DA, Jennings PB, Cooper JA (1996) Dynamics of capping protein and actin assembly in vitro: uncapping barbed ends by polyphosphoinositides. The Journal of cell biology 135: 169-179.

45. Delatour V, Helfer E, Didry D, Le KHD, Gaucher JF, et al. (2008) Arp2/3 controls the motile behavior of N-WASP-functionalized GUVs and modulates N-WASP surface distribution by mediating transient links with actin filaments. Biophysical journal 94: 4890-4905.

46. Matzavinos A, Othmer HG (2007) A stochastic analysis of actin polymerization in the presence of twinfilin and gelsolin. Journal of theoretical biology 249: 723-736.

47. Gillespie DT (1976) A general method for numerically simulating the stochastic time evolution of coupled chemical reactions. Journal of computations physics 22: 403-434. 


\section{Figure Legends}

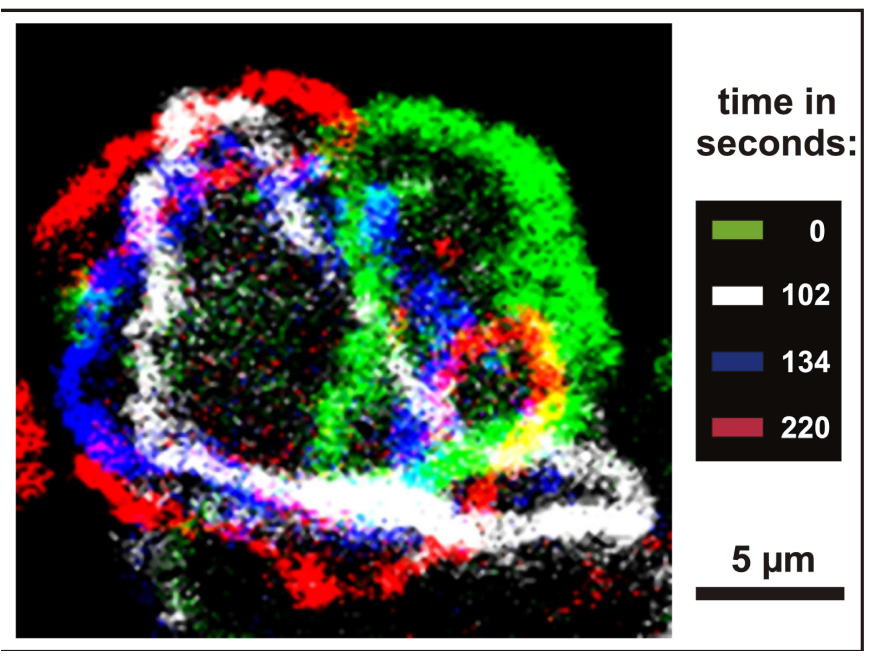

Figure 1. TIRF snapshots of actin wave dynamics in Dictyostelium discoideum. The colored bands denote the actin density at the indicated times. (From [16] with permission.)

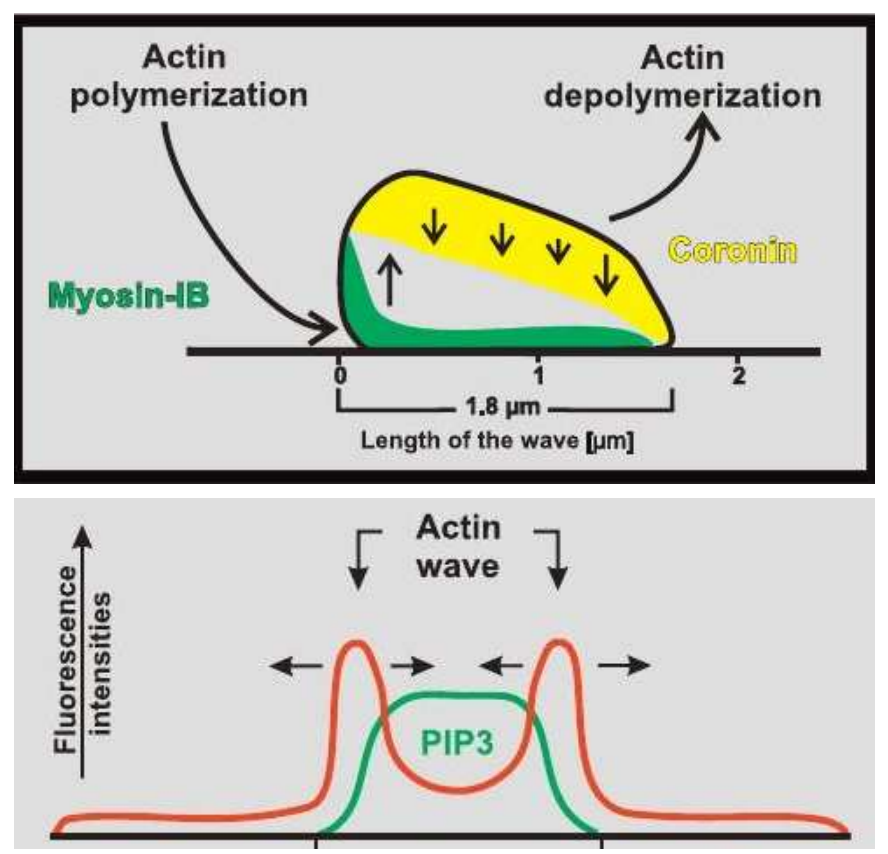

Figure 2. (Top) A cross-sectional view of the actin network within a wave, showing net polymerization at the front and net depolymerization at the top and rear. (Bottom) The distribution of actin and $\mathrm{Pip}_{3}$ in a cross-section of a wave. (From [9], with permission.) 


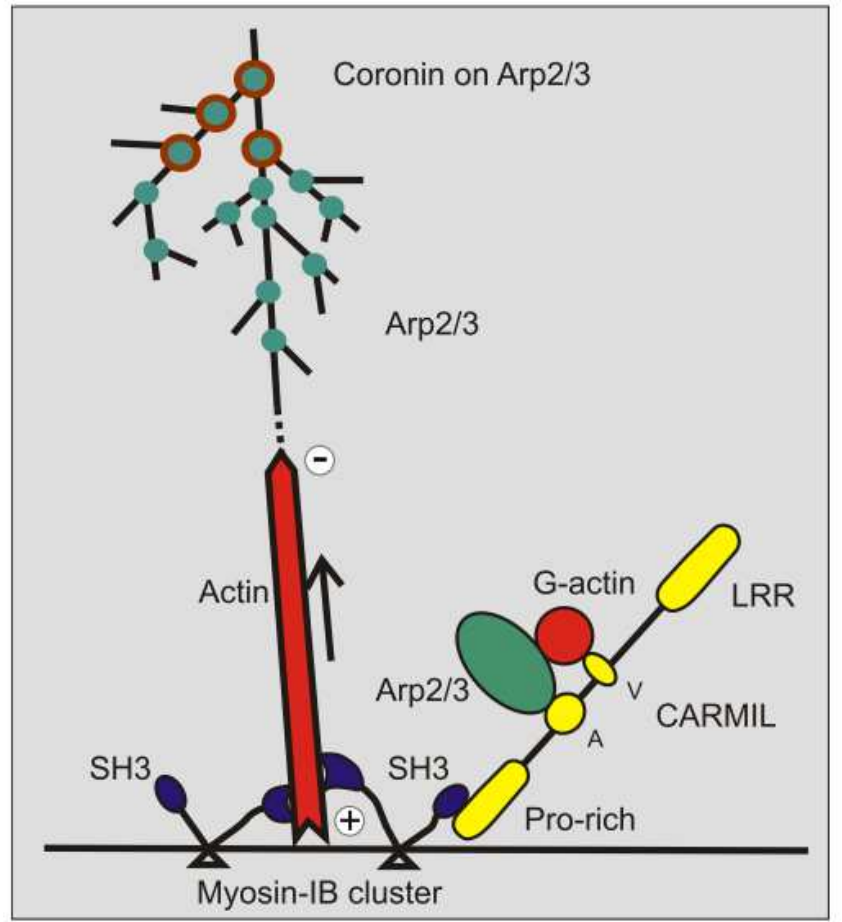

Figure 3. A schematic of a suggested model for actin wave formation. The tail of Myo-IB (blue) binds to the plasma membrane while the motor attempts to move toward the plus end of an actin filament, which maintains attachment of the growing filament to the membrane. The head may also attach to the scaffold protein CARMIL (yellow), which links it to the Arp2/3 complex, where new branches are formed via Arp2/3 binding (green). The activity of the Arp2/3 complex is inhibited by coronin (brown circles). (From [9] with permission). 


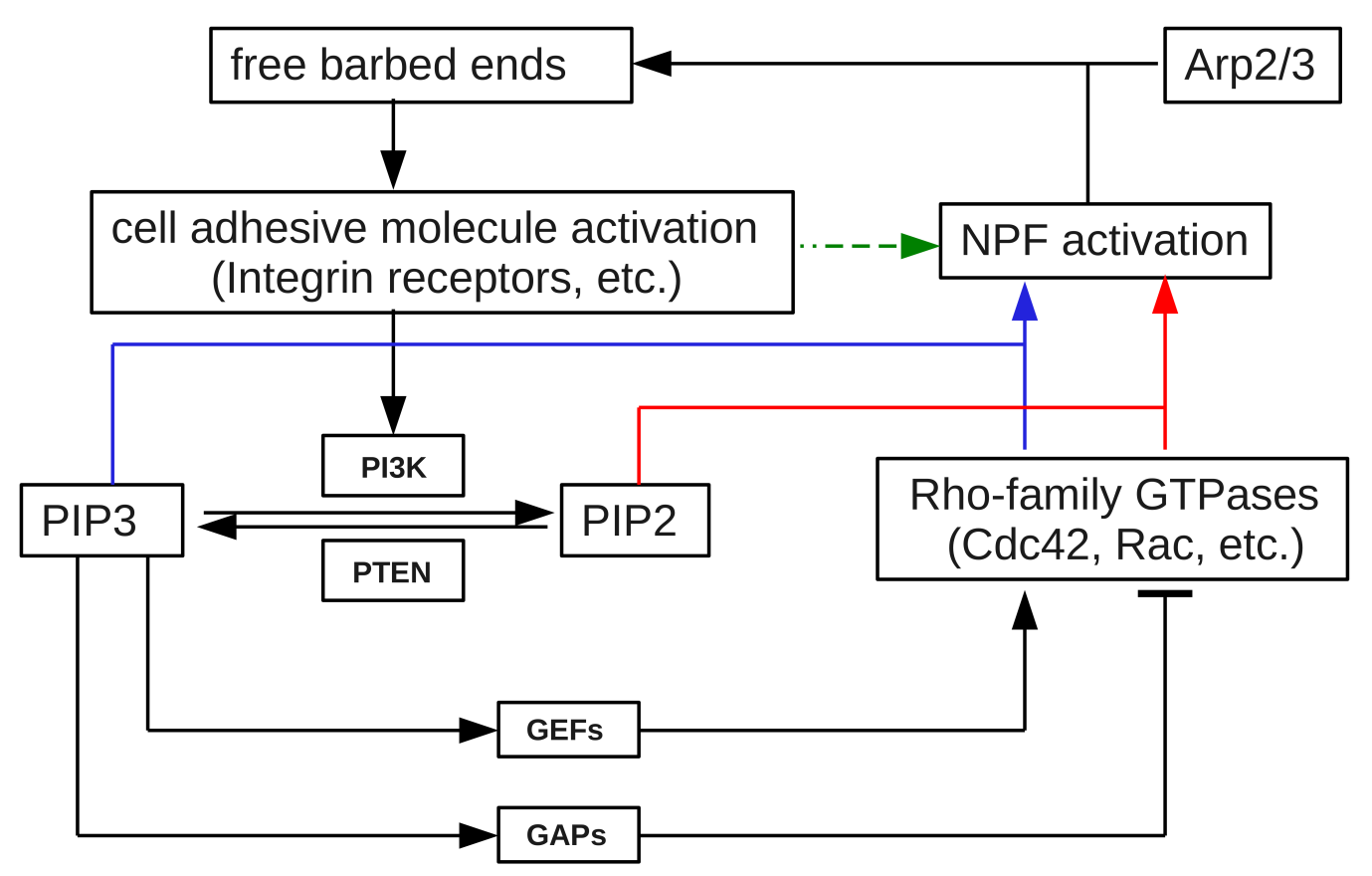

Figure 4. The feedback loop interactions between F-actin polymerization and signaling on the membrane. Note there are two ways cell adhesion molecules (CAM) induce F-actin polymerization: CAM may activate NPF and Arp2/3 at early and transient stages via FAK and/or vinculin (green dashed pathway), or CAM may activate NPF through the PI3K/PIP $3 /$ Rho-family GTPases cascade (red and blue pathways). 


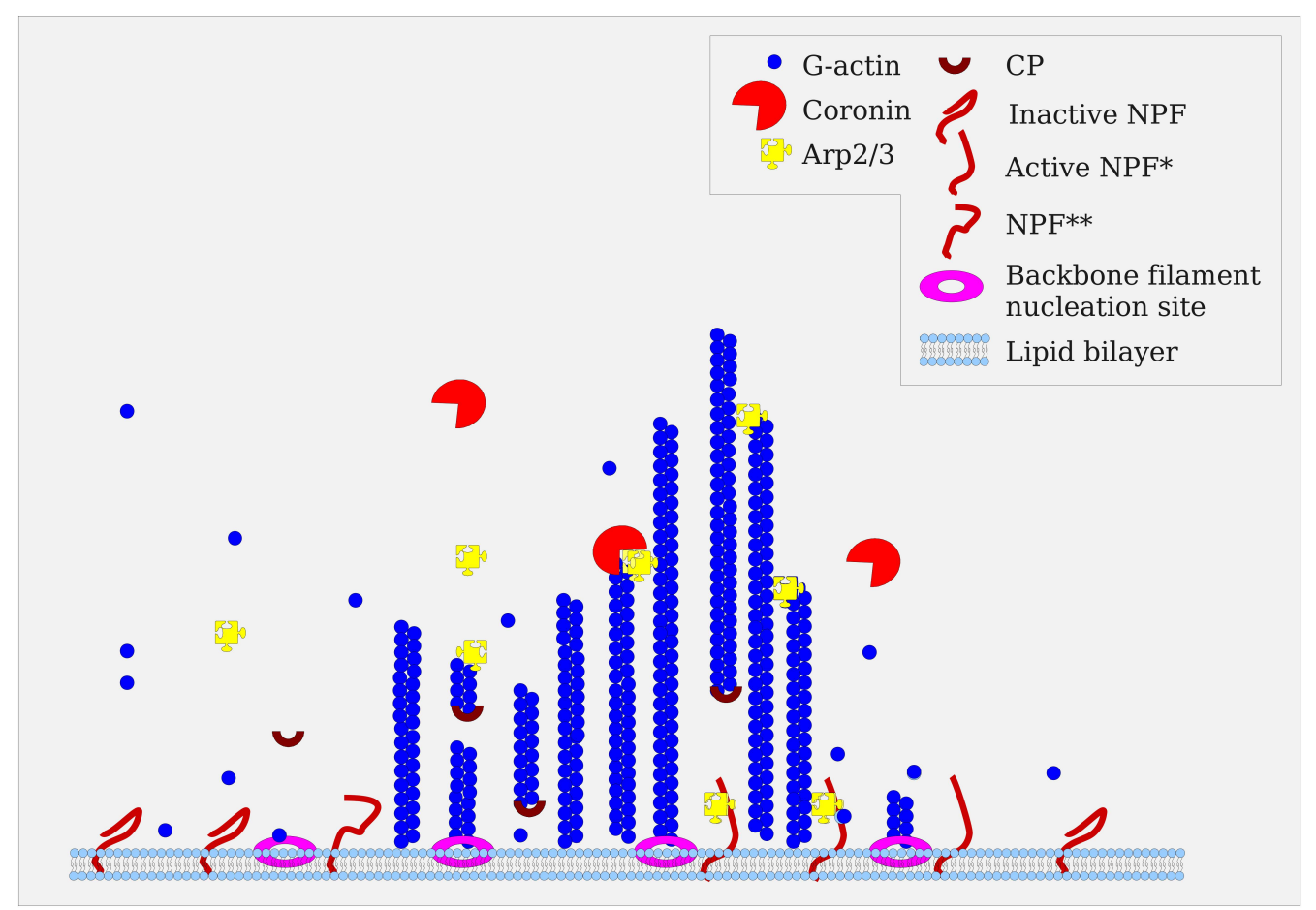

Figure 5. A schematic of the stochastic model for actin wave formation and propagation analyzed herein. Nucleation sites for the formation of backbone filaments, which are generated by G-actin attached to the site, reside on the membrane. Active NPF* can recruit Arp2/3 complex and G-actin to the membrane, and when the trimeric complex binds to the side of a filament, it generates a new filament branch with its pointed end capped and linked to the mother filament. The cytoplasmic coronin may bind to Arp2/3 complex at the branch junction, and remove it from the branch site and expose the filament pointed end. Free barbed ends in close proximity to the membrane are assumed to activate inactive NPF, and after branch generation $\mathrm{NPF}^{*}$ is converted to $\mathrm{NPF}^{* *}$, which in turn slowly recovers to NPF. 


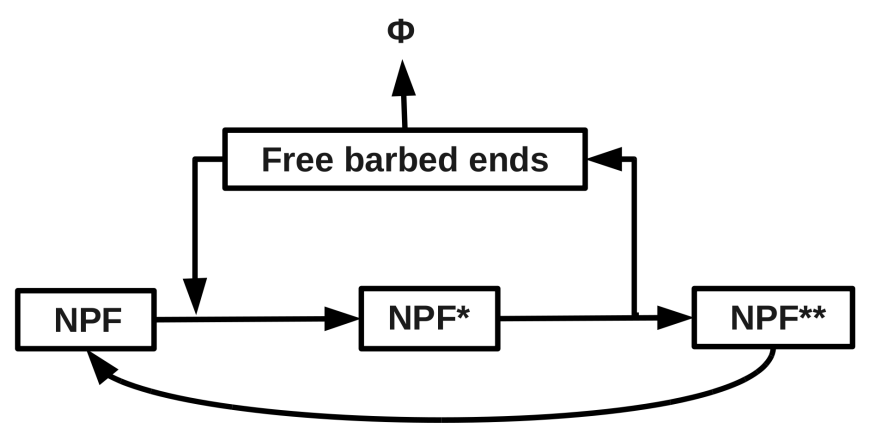

Figure 6. The simplified feedback loop between free barbed ends and various NPF states. $\mathrm{NPF}$ is activated to NPF* by free barbed ends of branched filaments, which in turn promotes barbed end generation and itself becomes inactive $\mathrm{NPF}^{* *}$. NPF** slowly recovers to inactive NPF. Free barbed ends can be capped and the filament can depolymerization completely. 
bioRxiv preprint doi: https://doi.org/10.1101/2019.12.31.892034; this version posted December 31, 2019. The copyright holder for this

preprint (which was not certified by peer review) is the author/funder, who has granted bioRxiv a license to display the preprint in perpetuity. It is made available under aCC-BY-NC 4.0 International license.
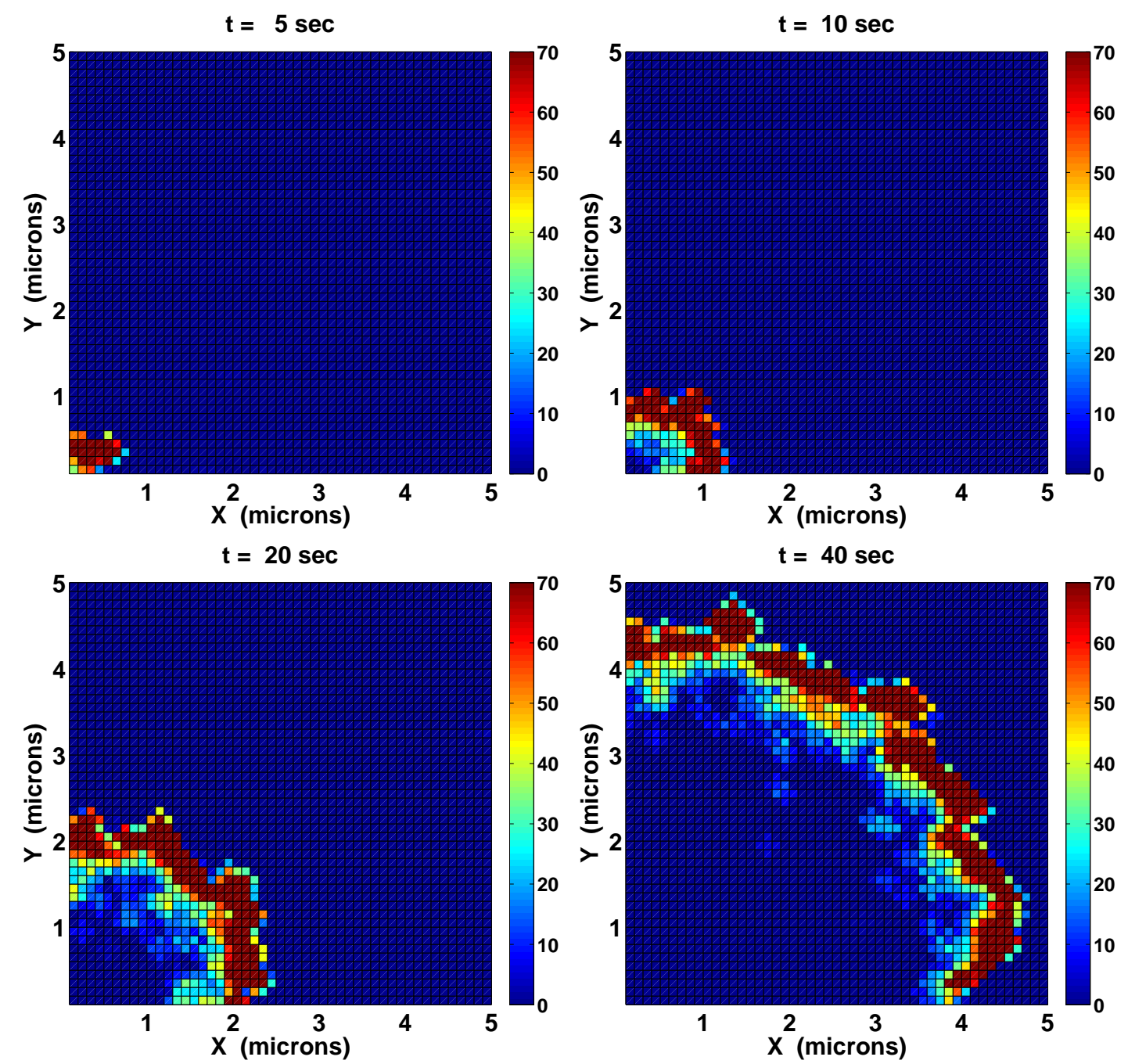

Figure 7. A computational TIRF sequence for the formation and propagation of an F-actin wave. The initial G-actin concentration is $10 \mu \mathrm{M}$, and half of the NPF is activated at the lower left corner. The color index indicates the total F-actin within $100 \mathrm{~nm}$ of the membrane projected to each membrane compartment. The maximal density in the color representation is set to be 70 monomers per compartment, and thus density larger than 70 is colored the same as 70 . However, the highest density of F-actin throughout the membrane could be larger than 70 . 


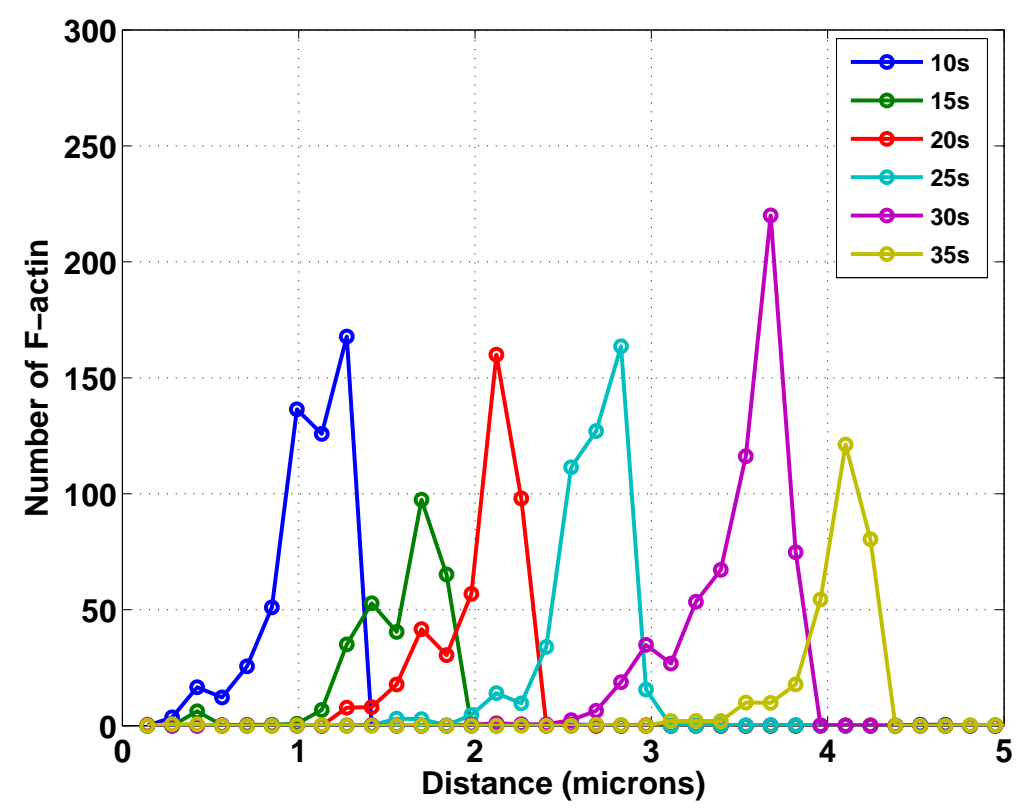

Figure 8. The propagation of an F-actin wave. This 1D description of wave dynamics is derived from a diagonal cross-section of the F-actin density shown Fig . 7.

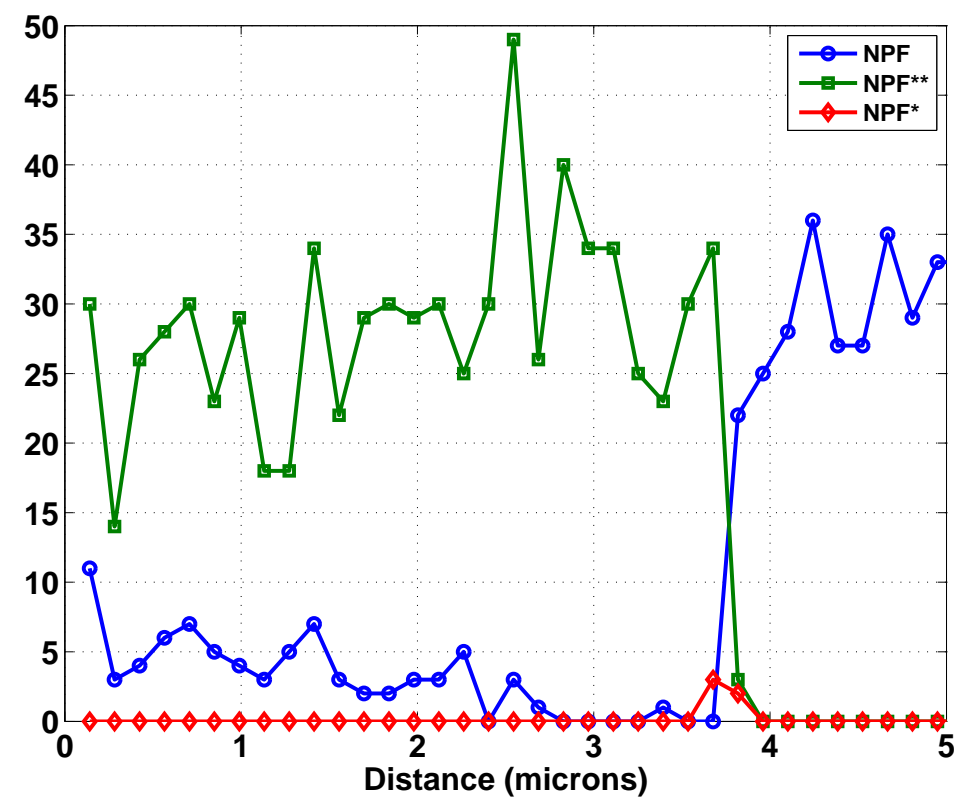

Figure 9. The distribution of various NPF states at 30 seconds of the wave dynamics. This distribution is along a cross-line through the wave on the $\mathrm{x}-\mathrm{y}$ plane as in Fig. 8. The y-axis is the density $\left(\# / 0.01 \mu m^{2}\right)$ of various NPF's. 

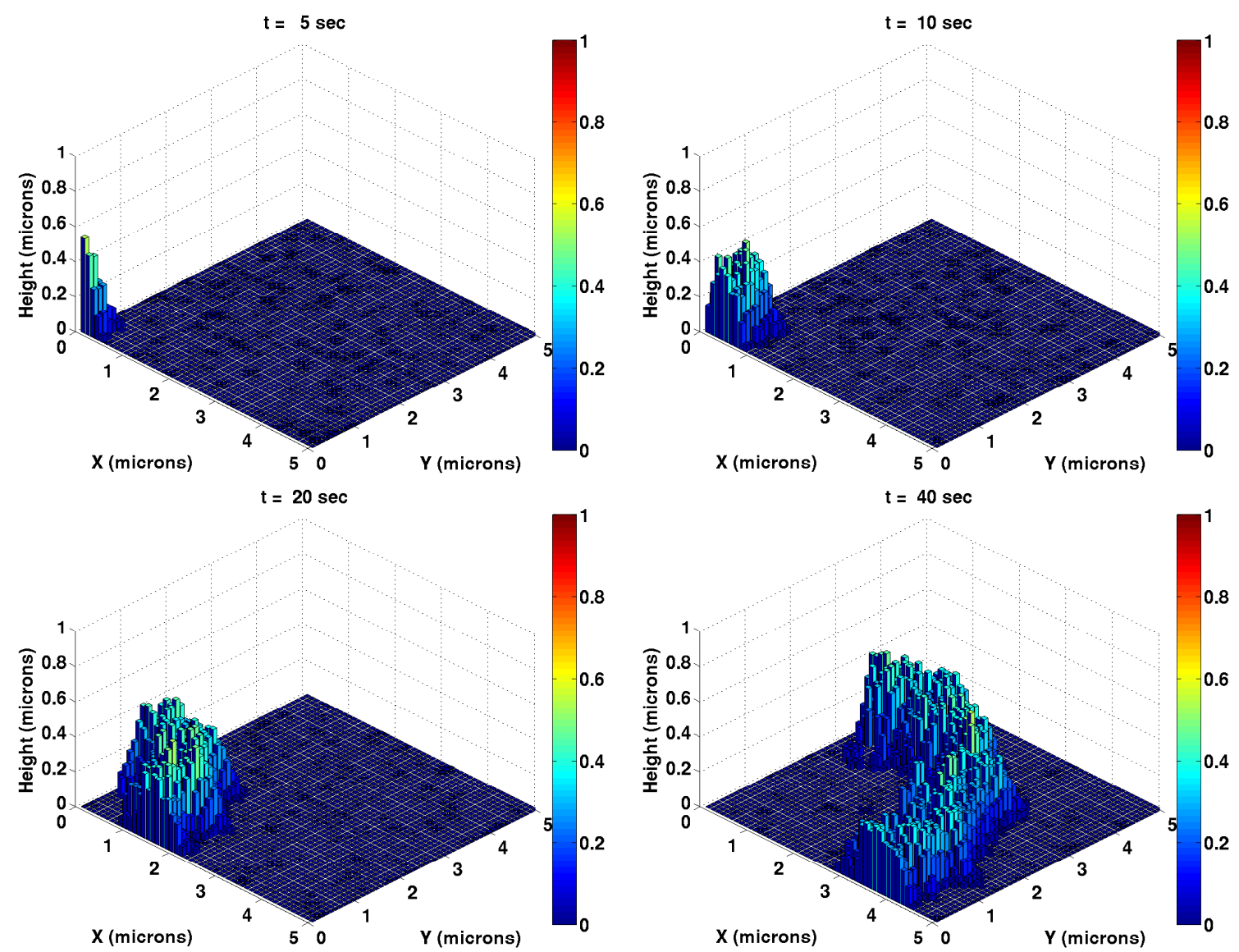

Figure 10. A 3D depiction of the temporal evolution of a wave. The color-coded solid bars show the maximal height of the actin network at the corresponding computational cell on the membrane. 

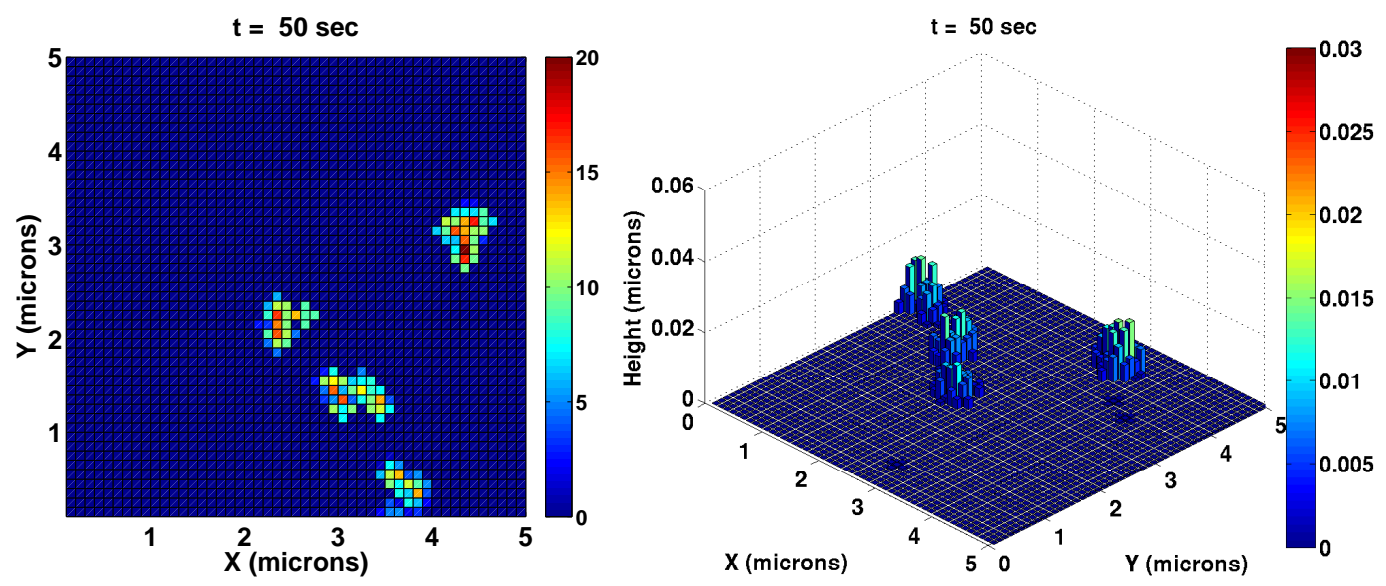

Figure 11. The TIRF image of F-actin and height profile for actin spot formation at low $0.1 \mu \mathrm{M}$ G-actin concentration.

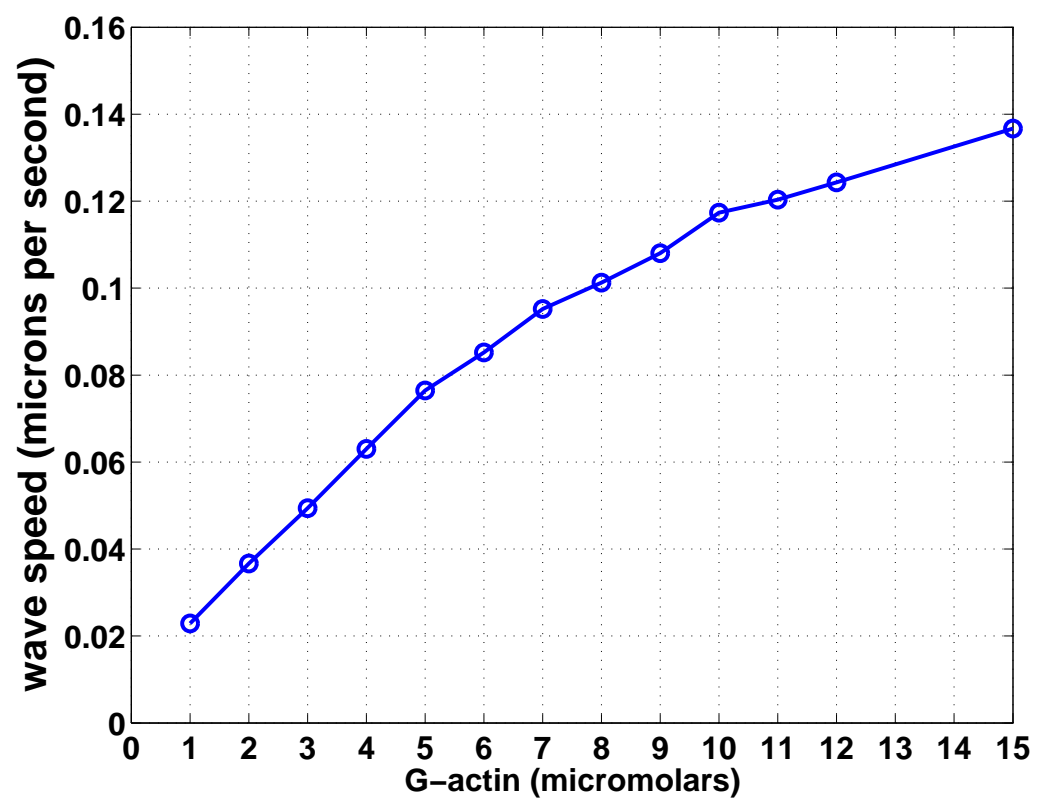

Figure 12. The dependence of the wave speed on the actin monomer concentration. The average speed for twenty realizations is shown at each concentration. For each realization, the distances from points along the wave front to the initial NPF activation site are tracked at each time point in order to perform proper statistical analysis. 

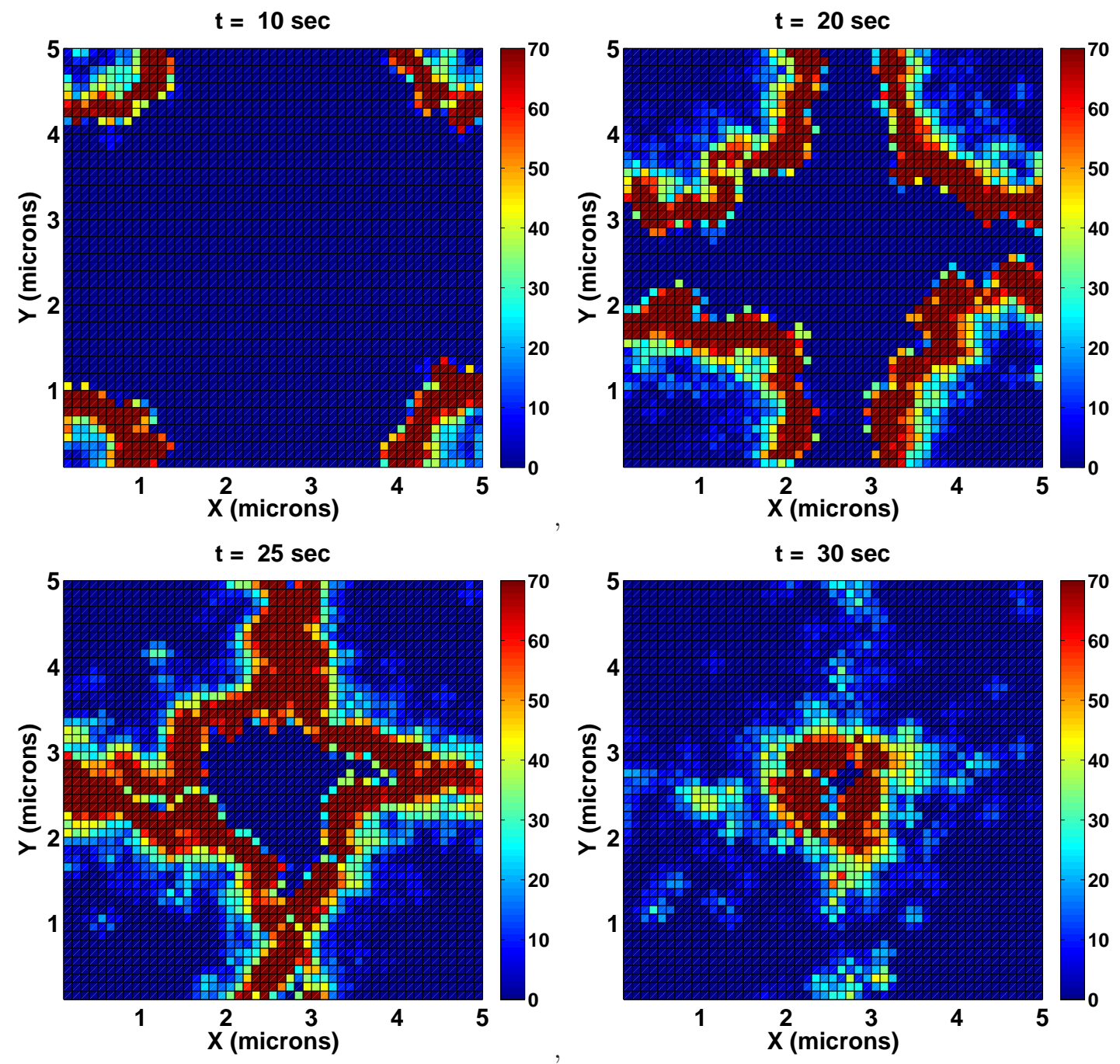

Figure 13. TIRF images of colliding actin waves. The NPF is partially activated at four corners at the domain. 

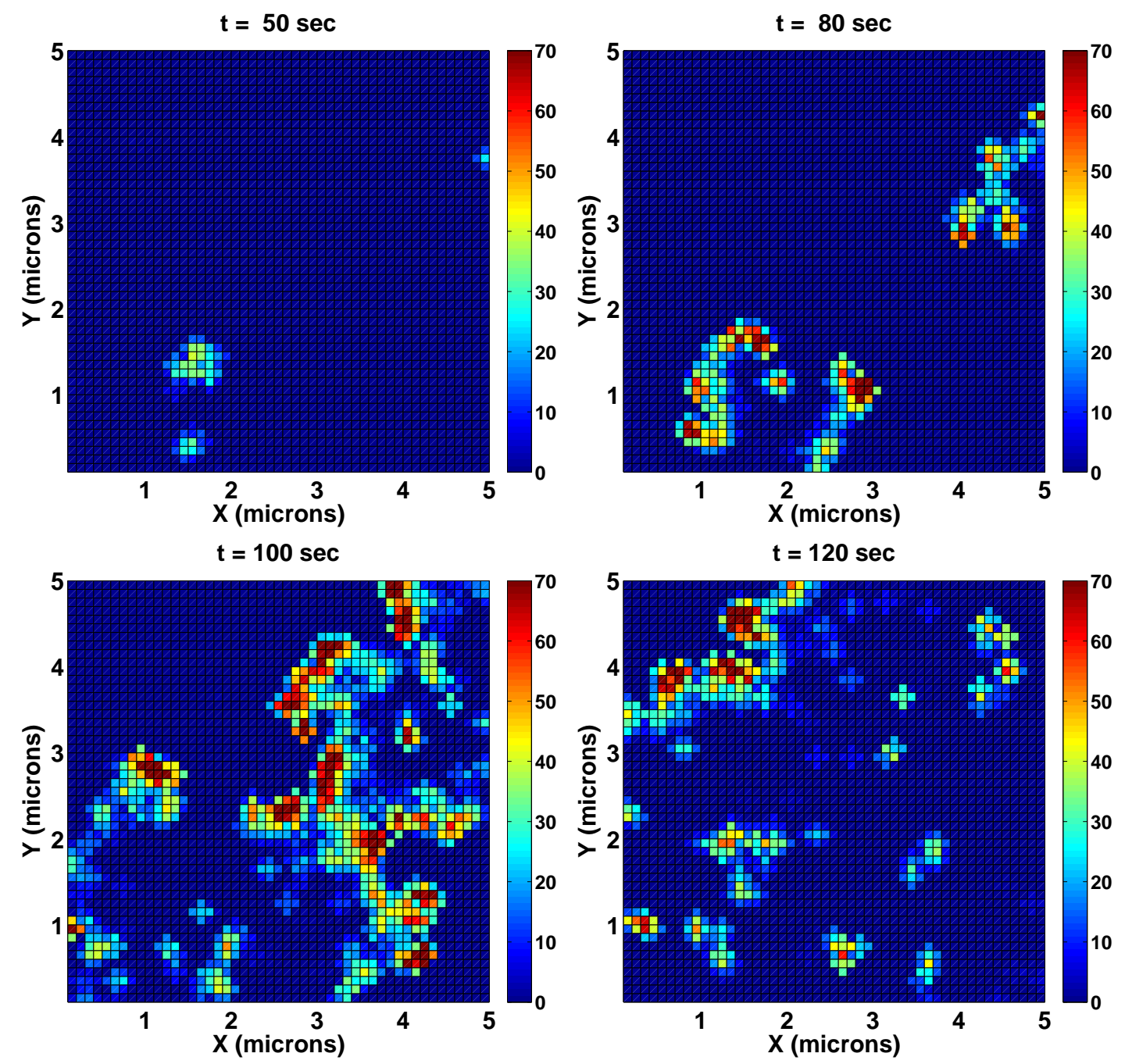

Figure 14. TIRF images of long term dynamics after the collision of waves. Note that three actin clusters remain on the membrane - two at the lower left and one at the upper right - at $t=50.0$ seconds. The dynamics shown are the continuation of those in Fig. 13. 

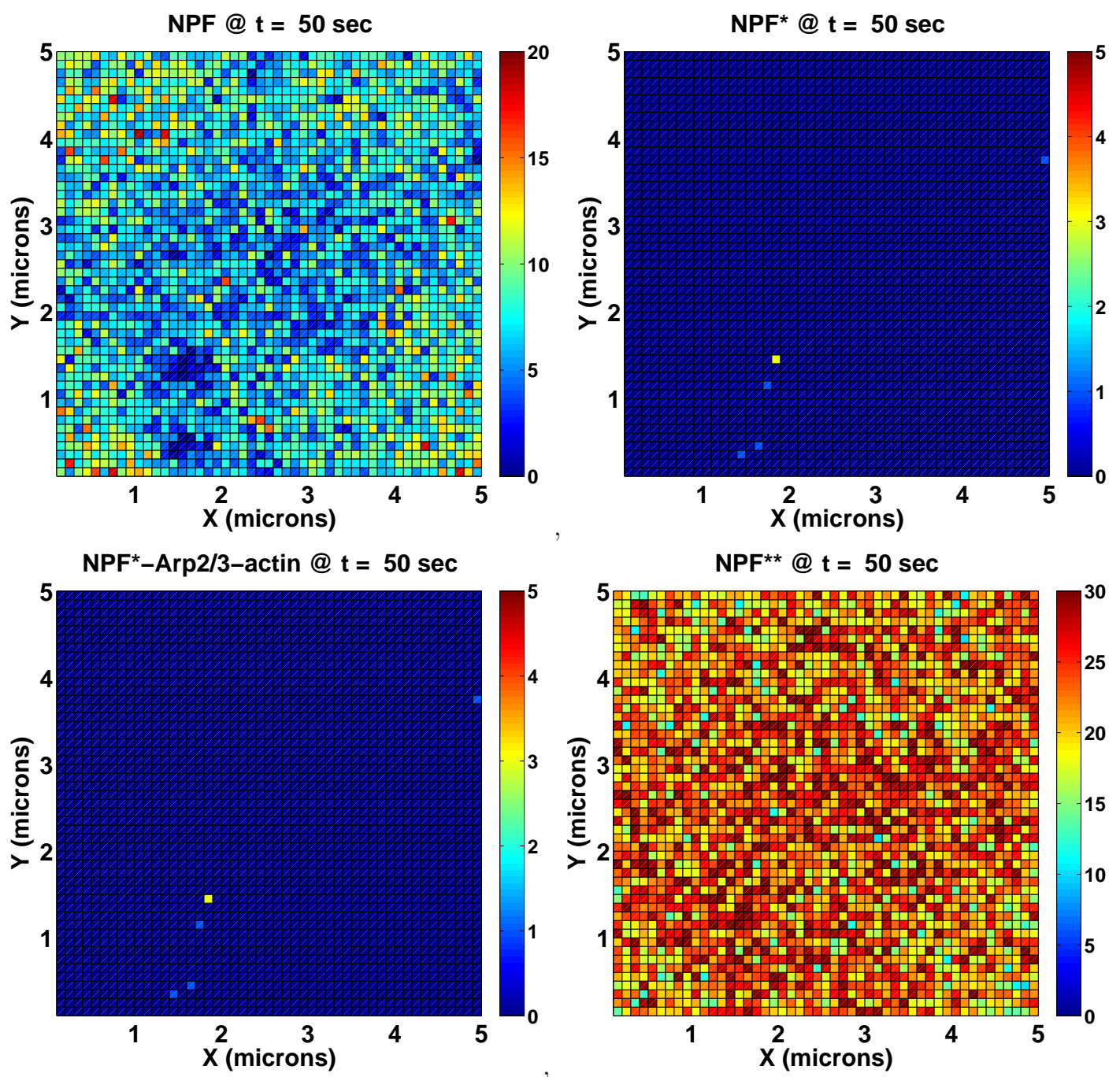

Figure 15. The distribution of various NPF states at $t=50.0$ seconds for the waves shown in Fig. 14. Note that at this time a significant portion of $\mathrm{NPF}^{* *}$ has recovered to NPF, which is able to generate new waves with free barbed end seeds. 

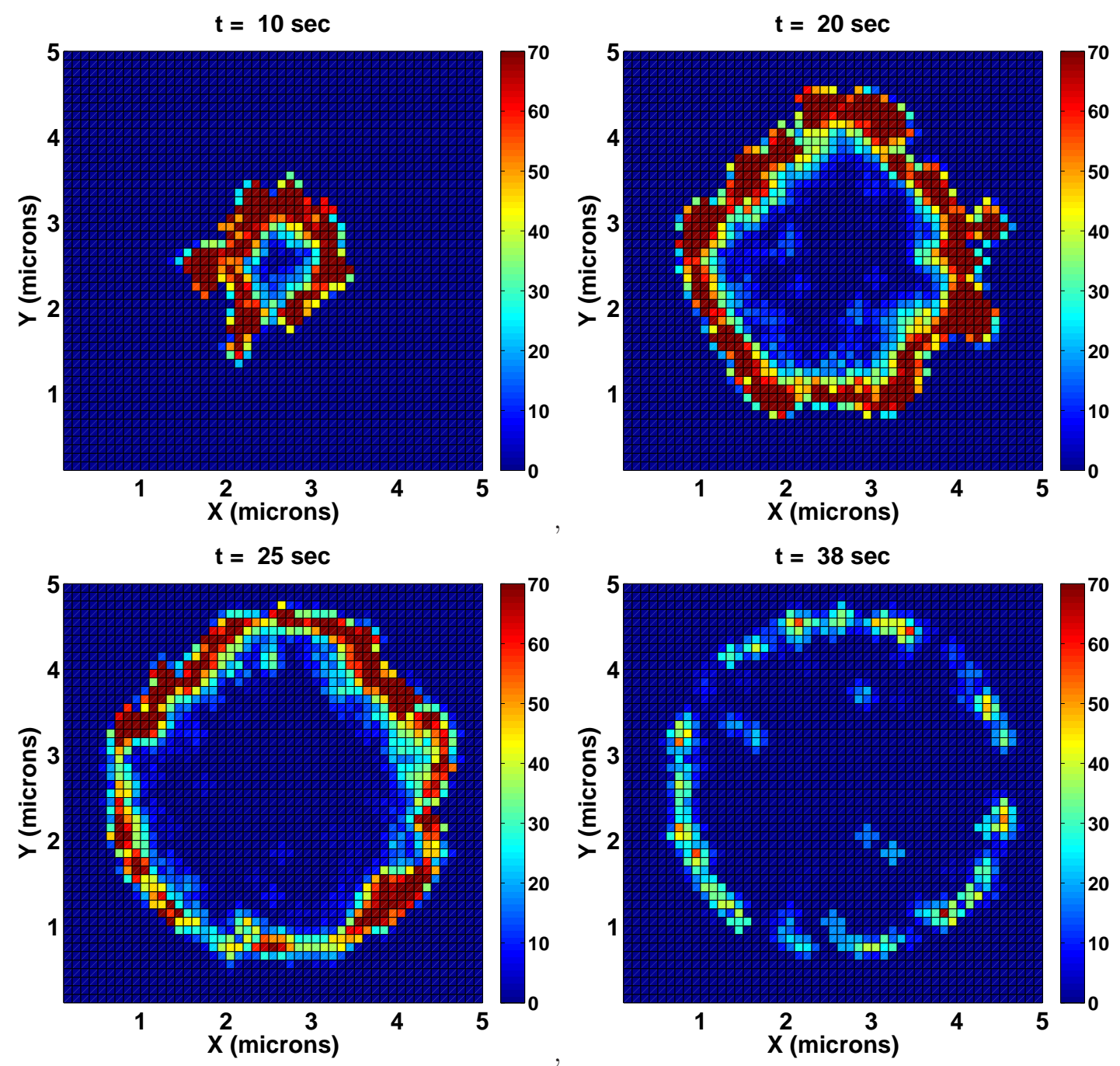

Figure 16. The standing wave at the border of attached and unattached membrane. 

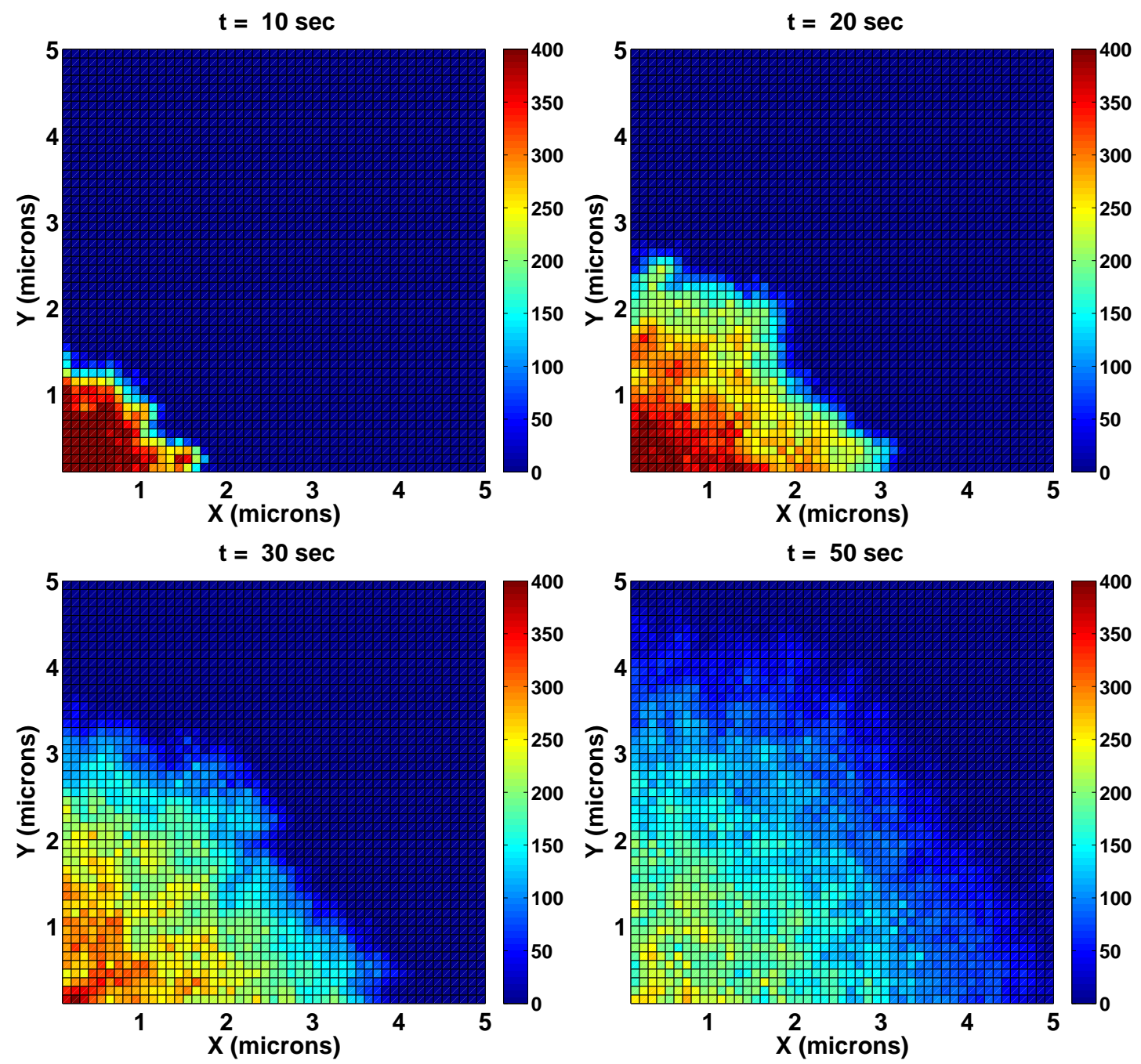

Figure 17. TIRF images of F-actin dynamics for the model based on two NPF states. In this model NPF* immediately becomes NPF after successfully generating a new barbed end. Note that the F-actin density scale here is different from that in Fig. 7. 

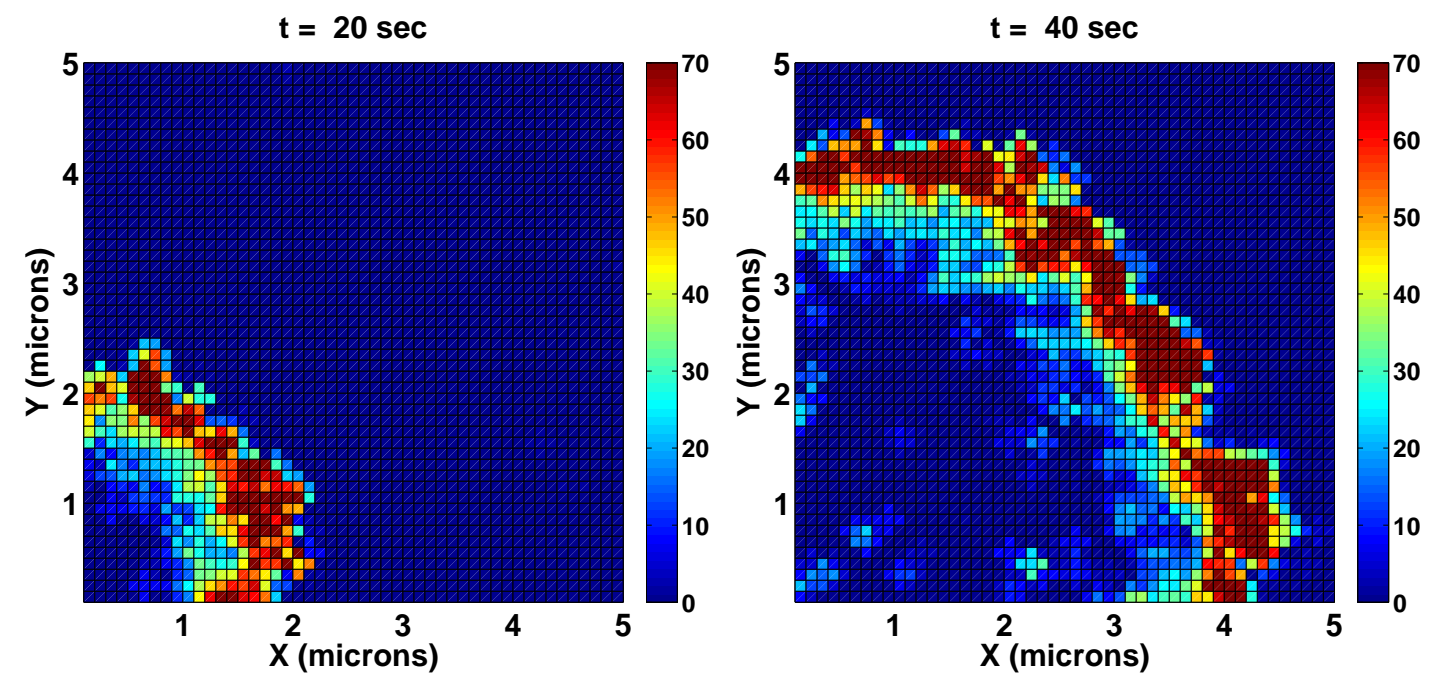

Figure 18. TIRF images of an F-actin wave when NPF is allowed to detach from the membrane and diffuse in the cytosol before re-attaching to the membrane.

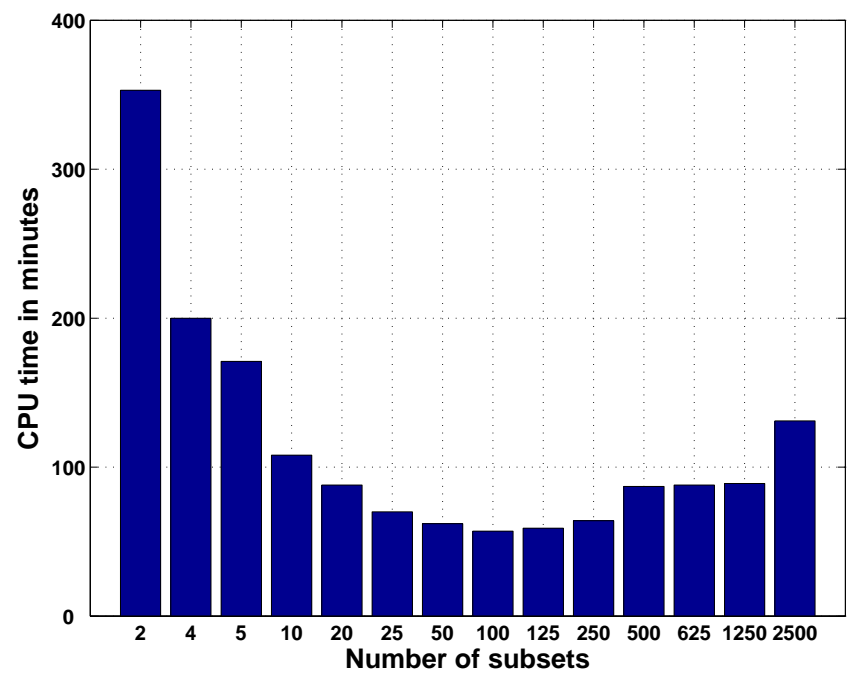

Figure 19. The CPU times taken for the simulation with various subdivision sizes for the compartments. The CPU time is the time required for computing the first 5 seconds of the dynamics shown in Fig. 7. Without subdividing the compartments, the computation takes about 750 minutes. 


\section{Supporting Information}

\section{Video S1}

3 Time evolution of wave birth and propagation. The wave was initiated with NPF activation at the

4 left bottom corner at $t=0$ second. The F-actin density in each simulating compartment is the F-actin

5 within a $100 \mathrm{~nm}$ depth of the cytoplasm onto the membrane, which is to be compared with TIRF images

6 of the wave. The condition of this realization of the stochastic model is the same as that in Fig. 7 .

\section{Video S2}

8 Time evolution of the network shape toward the cytoplasm. The bar in each compartment

9 represents the height of the filament pointed end which situates farthest from the membrane above that

10 compartment.

${ }_{11} \quad$ Video S3

${ }_{12}$ Time evolution of the network shape toward the cytoplasm. The NPF's are activated at the 13 center of the membrane plane.

${ }_{14}$ Video S4

15 F-actin wave dynamics with NPF cycling between the cytosol and membrane.

\section{${ }_{16}$ Video S5}

17 The dynamics of wave collision and subsequent new wave formation. 